\title{
Sono solo canzonette? L'emancipazione sessuale nella canzone leggera italiana degli anni Settanta
}

"Sono solo canzonette? " L'émancipation sexuelle dans la musique de variété italienne des années soixante-dix

\section{Licia Bagini}

\section{(2) OpenEdition}

\section{Journals}

Edizione digitale

URL: http://journals.openedition.org/cei/1210

DOI: 10.4000/cei.1210

ISSN: 2260-779X

\section{Editore}

UGA Éditions/Université Grenoble Alpes

Edizione cartacea

Data di pubblicazione: 30 juin 2013

Paginazione: 157-200

ISBN: 978-2-84310-245-5

ISSN: 1770-9571

\section{Notizia bibliografica digitale}


SONO SOLO CANZONETTE?

\author{
L'EMANCIPAZIONE SESSUALE NELLA CANZONE \\ LEGGERA ITALIANA DEGLI ANNI SETTANTA
}

\author{
Licia Bagini \\ Université de Poitiers
}

Questo articolo si propone di analizzare la presenza e le modalità di rappresentazione dell'identità sessuale nelle canzoni leggere italiane degli anni Settanta. Se osservati rapidamente, i brani in voga negli anni Settanta mostrano una prevalenza di stereotipi diffusi sui comportamenti uomodonna nella società del tempo, spesso in décalage evidente con le mutazioni sociali in atto. Tuttavia, una lettura testuale più attenta fa spesso apparire riferimenti a comportamenti e identità sessuali meno 'tradizionali'.

Un primo problema incontrato a livello metodologico è stato quello della scelta dei brani a cui fare riferimento. Come procedere a una selezione di canzoni nella ricca e svariata produzione discografica di quegli anni? Dopo aver interrogato un centinaio di persone tra i 50 e 70 anni — di ambo i sessi, d'estrazione sociale e professioni differenti - a proposito delle canzoni italiane degli anni Settanta che preferivano o che ricordavano in particolare per motivi diversi, ho voluto verificare se le canzoni indicatemi corrispondessero a quelle più vendute o premiate. Mi sono allora imbattuta in nuove difficoltà, perché non sempre le due cose coincidevano. Per esempio, non sempre la canzone premiata a un certo festival era quella più ricordata o venduta.

Alla fine, ho adottato il criterio seguente: prendere le prime dieci canzoni italiane più vendute degli anni Settanta ${ }^{\mathrm{I}}$, indipendentemente da

I. È possibile accedere alle classifiche dei maggiori successi musicali consultando il sito <www.hitparadeitalia. it/indici/per_anno/hpyi970.htm> (data di ultima consultazione 8 dicembre 20I2). L'anno indicato per le canzoni corrisponde alla loro più lunga permanenza nella hit parade, che non coincide necessariamente con il loro anno d'uscita. Il numero della posizione in classifica è relativo solo alla lista delle canzoni italiane dal 1970 al I979, senza che qui si tenga conto dei pezzi stranieri. Sottolineo nondimeno che, nelle hit parade degli anni 
considerazioni legate alle ragioni del loro successo, e analizzarne alcune particolarmente emblematiche rispetto alla tematica del volume. Questo corpus si basa quindi sui dati delle vendite delle classifiche annuali in cui ritroviamo vari generi musicali. Senza entrare in merito alla definizione di cosa s'intenda per musica leggera ${ }^{2}$, è ovvio che quando una canzone entra nelle classifiche di grande successo ha un impatto maggiore su un vasto pubblico di ascoltatori ${ }^{3}$. Non mancherò, però, di segnalare alcuni pezzi che hanno segnato un'epoca e che meritano, quindi, di essere ricordati, pur non comparendo nelle classifiche delle canzoni più vendute.

Per meglio inquadrare il periodo studiato, mi pare indispensabile ricordare (soprattutto per un pubblico giovane) che negli anni Settanta i dischi più venduti sono stati i 45 giri contenenti una canzone per lato, e che nella seconda metà di quel decennio i giovani cominciano a preferire i 33 giri - contenenti più pezzi - in cui si trovavano canzoni molto note non proposte nella versione 45 giri $^{4}$. Ricordo pure l'arrivo delle audiocassette che hanno facilitato la diffusione d'ascolto, perché, oltre alla facilità di trasporto, permettevano la registrazione delle canzoni preferite senza dover ricorrere all'acquisto dei dischi.

Per la mia analisi mi sono basata sulle canzoni più gettonate in versione single (cioè 45 giri) che, d'altra parte, si ritrovano pure nei 33 giri più venduti.

Gli anni Settanta sono quindi un periodo di grandi cambiamenti tecnologici, caratterizzato dall'utilizzazione di nuovi strumenti di ascolto e di diffusione delle canzoni come il mangiadischi, il registratore mangiacassette e i videoclip. I canali di diffusione aumentano e si sviluppano: ai festival e concorsi canori ${ }^{5}$ affermati da tempo si aggiungono nuove manifestazioni musicali dal vivo e concerti sempre più ritrasmessi sui canali televisivi e radiofonici della $\mathrm{RAI}^{6}$. Nella seconda metà degli anni Settanta,

Settanta, figurano poche canzoni straniere fra le prime dieci. In Italia, i successi dei 45 giri stranieri cominceranno ad affermarsi sul mercato discografico a partire dagli anni Ottanta, sorpassando la presenza di successi italiani.

2. Cfr. Franco Fabbri, Il suono in cui viviamo, Milano, Il Saggiatore, 2008, pp. I7-I8 e 67-99.

3. In Italia, la prima trasmissione radiofonica consacrata ai 45 giri più venduti nel paese va in onda a partire dal 1967 e porta il titolo di Hit Parade, termine che verrà sempre più utilizzato al posto dell'equivalente italiano "classifica». Le classifiche televisive iniziano più tardi (1977) con le trasmissioni Discoring e Superclassifica show.

4. I 45 giri, nati nel 1948, sono i vinili più diffusi fino agli anni Sessanta. I 33 giri — chiamati anche LP appaiono nel 1965; contenevano in media una decina di pezzi e resteranno in auge fino agli anni Ottanta. Le cassette coi nastri compatti arrivano nel 1965 e restano in voga fino agli anni Novanta. Saranno entrambi sostituiti dai CD (1982), quindi dall'MP3 (1993).

5. Tra i più noti ricordiamo il Festival di Sanremo, nato nel 1951, il Cantagiro nel 1962, il Festivalbar e Il Disco per l'estate lanciati nel 1964 .

6. In quegli anni era difficile sfuggire all'ascolto delle canzoni che studieremo, non solo perché la larga maggioranza delle famiglie disponeva di un televisore e di una radio, ma anche perché questi brani venivano 
cominciano poi a diffondersi le radio libere, seguite rapidamente dalle reti televisive private. Questo fenomeno è interessante per diverse ragioni, in quanto le radio libere si rivolgevano a un pubblico di ascoltatori più giovane, portatore di nuovi ideali e spesso meno conformista dei genitori, e trasmettevano canzoni censurate dalla RAI. Non sarà una sorpresa ricordare che la censura di «mamma RAI» colpiva essenzialmente testi di canzoni che facevano riferimento in termini troppo espliciti alla sessualità, e ciò malgrado si stesse attraversando un periodo d'apertura ed emancipazione in questo campo.

Mi sono soffermata in particolare sui testi, ma evidentemente il successo di una canzone è indissolubilmente legato alla musica. Basti pensare a successi inglesi o americani amati da un pubblico che non capisce necessariamente tutte le parole. Non essendo musicologa e per ragioni di spazio, non farò che qualche accenno alla melodia, ma è ovvio che leggere un testo senza ascoltare la musica che l'accompagna è una cosa completamente diversa. Questo vale in parte anche per quanto riguarda l'ascolto della canzone da sola o accompagnata dall'immagine dell'interprete che la esegue. L'identificazione tra il soggetto cantante e l'oggetto cantato è essenziale, tant'è vero che la musica e l'interpretazione possono far dimenticare o maggiormente apprezzare le parole di una canzone. A questo bisogna aggiungere il fatto che, come ricorda Enzo Gentile: «Il look, un certo culto dell'immagine, servirà non poco dagli anni Settanta in poi, quando i cantanti che lo desiderano avranno dalla loro parte i giornali, le riviste specializzate, il mercato dei poster e quindi i video-clip.» ${ }^{7}$

È proprio a partire dagli anni Settanta che la musica leggera tocca un pubblico sempre più grande e differenziato, diventando un fenomeno commerciale non trascurabile ${ }^{8}$.

ampiamente diffusi in luoghi pubblici (supermercati, bar, spiagge). Come afferma Giuseppe Antonelli, per gli italiani "già nei primi anni Sessanta quello delle canzonette poteva essere descritto come un 'rumore quotidiano’ presente ovunque» (Giuseppe Antonelli, Ma cosa vuoi che sia una canzone, Bologna, Il Mulino, 20Io, p. 86).

7. Enzo Gentile, Legata a un granello di sabbia, Milano, Melampo, 2005, p. 8. La diffusione di videoclip resta comunque molto timida in questo periodo.

8. «[...] oltre a un'estensione generale dell'interesse per la musica [...] il fatturato dell'industria discografica italiana, dal I970 al 198I, più che quintuplica, da 44 a 230 miliardi di lire» (Enzo Gentile, Legata a un granello di sabbia, cit., p. 8). 


\section{Corpus}

Essendo il nostro $\operatorname{corpus}^{9}$ composto dai pezzi più venduti negli anni Settanta, le nostre osservazioni portano essenzialmente sulle cosiddette "canzoni di consumo" o "canzoni commerciali», terminologia che viene sempre più usata a partire dal periodo in questione in opposizione alle "canzoni d'autore», ritenute di più alto valore e spesso eseguite da chi ne aveva scritto parole e musica ${ }^{\mathrm{I}}$. Le canzoni commerciali erano raramente interpretate da cantautori, e ancor meno da cantautrici. Notiamo anche che, tra le prime dieci canzoni delle classifiche, troviamo più cantanti uomini che donne, e che tra i grandi degli anni Settanta dominano la scena Lucio Battisti - le cui canzoni sono indissociabili, in questo periodo, dal paroliere Mogol - e Mina. Progressivamente entreranno nelle hit parade i complessi, composti prevalentemente da uomini.

Nella selezione delle canzoni studiate ${ }^{\mathrm{II}}$ cercheremo di mettere in parallelo alcuni successi che veicolano stereotipi dei ruoli uomo-donna presenti nella società italiana di quel periodo con altri nei quali affiorano immagini più innovative, a volte persino sorprendenti, testimonianza di un periodo di contraddizioni e grandi cambiamenti nella società.

\section{L'infedeltà maschile}

Il soggetto più diffuso nelle nostre canzoni è l'amore, spesso accompagnato dal tema del tradimento. In Italia - dove il divorzio, dopo lunghi anni di dibattiti, era stato legalizzato nel 1970, per essere poi rimesso in questione, tanto da arrivare al referendum del 1974 - prevale una concezione tradizionale della coppia fondata sulla fedeltà, sostenuta evidentemente dalla Chiesa. Nonostante questo, moltissime sono le canzoni i cui testi raccontano tradimenti riferiti, soprattutto, all'uomo nel ruolo di

9. Cfr. Allegato I, p. I79.

IO. Il termine «cantautore» comincia a entrare timidamente nei cataloghi discografici negli anni Sessanta davanti ai nomi, per esempio, di Gino Paoli, Luigi Tenco, Giorgio Gaber o Fabrizio De André. Tuttavia la figura del cantautore, visto come una sorta di 'vate' che affronta e denuncia problemi sociali con testi 'impegnati' e ricorrendo a uno 'stile più alto', nasce proprio negli anni Settanta con l'arrivo di altri cantautori, come, per esempio, Francesco De Gregori, Edoardo Bennato, Roberto Vecchioni, Francesco Guccini. Questi cantautori troveranno poi riduttiva questa categoria e la criticheranno aspramente nelle loro stesse canzoni (cfr. L'avvelenata di Guccini o Cantautore di Edoardo Bennato, entrambe del 1976). Ricordiamo pure che le cantautrici erano molto più rare dei cantautori.

II. Questa selezione, puramente personale, non si vuole certamente esaustiva. Si tratta piuttosto di una pista di riflessione che meriterebbe di essere sviluppata in futuro. 
'traditore'. Questo comporta, naturalmente, anche un discorso 'di riflesso' sul ruolo della donna nella coppia.

Prendiamo, per esempio, Tanta voglia di lei (Facchinetti-Negrini) ${ }^{\mathrm{I2}}$ dei Pooh, canzone melodica, accompagnata da pianoforte e violino, giunta seconda nella hit parade del 1971, che ha consacrato la fama di questo gruppo canoro. Il protagonista della storia, dopo aver passato la serata a casa di una donna (chiari sono i riferimenti al tipo di serata: «Mi dispiace di svegliarti», "morderai le lenzuola»), capisce che la persona che più conta per lui è la moglie o la 'compagna'. Rivolgendosi all'amante, egli afferma ipocritamente («forse un uomo non sarò») di provare dispiacere nell'andar via, ma il dovere lo spinge a tornare a casa ${ }^{13}$. Il rapporto con la prima donna si riassume a una rapida avventura — «strana amica di una sera» - a cui porre fine senza tanti ripensamenti — «chiudo gli occhi un solo istante/ la tua porta è chiusa già». Emerge quindi l'immagine di una donna 'usa e getta', opposta, senz'alcuna ambiguità, a quella della vera compagna, definita «il mio amore». Il ruolo dell'uomo è qui strettamente legato al motivo sessuale, in quanto dice di dover correre a casa perché ha «tanta voglia» della sua compagna, e chi, se non lui, «la scalderà»? In una sorta di autolusinga, quest'uomo si sente sessualmente indispensabile alle donne che lo frequentano, che nel testo risultano essere alla sua mercé. Se tutto questo corrisponde alla tradizionale immagine di macho allora comunemente diffusa, dobbiamo però notare che la Lei della canzone non è esplicitamente connotata come «moglie». Nonostante ciò, il fatto che venga chiamata «il mio amore» le dà una connotazione implicita di legittimità, e, per questo, la sessualità tra la coppia può esser evocata più esplicitamente, in quanto approvata dalla morale comune («la sua mano dolcemente cerca me»; "sta abbracciando il suo uomo»). D'altra parte, queste ultime parole riflettono una certa apertura delle mentalità: si parla di più della sfera intima di una coppia e, malgrado lo si faccia ricorrendo a metafore e altre figure retoriche, cominciano ad apparire termini più concreti o diretti. Per esempio, il termine «uomo» viene introdotto progressivamente nella lingua quotidiana degli anni Settanta accanto al termine «marito» ${ }^{14}$. Questo slittamento semantico rinvia a un'immagine più sessuata dell'individuo rispetto al termine «marito», che evoca invece uno

I2. I nomi tra parentesi che seguono i titoli delle canzoni indicano in ordine gli autori delle parole e della musica.

13. "Mi dispiace di svegliarti [...] so che devo lasciarti / il mio posto è là, il mio amore si potrebbe svegliare.»

I4. Lo stesso vale per «donna» al posto di «moglie». È pure vero che i termini «uomo»e "donna» — presi in quest'accezione di «marito» e "moglie» - erano già usati in molti dialetti, ma non bisogna vedere la loro diffusione nella lingua italiana di quel periodo come influenza dialettale. 
statuto ufficialmente riconosciuto dalla società. Il fatto che il protagonista si definisca «il suo uomo» proprio quando parla della compagna che cerca con dolcezza il corpo di lui risulta pertanto significativo a questo proposito.

In Anna (Mogol-Battisti), classificata al quarto posto nella hit parade del 1970, il protagonista ha decisamente un altro atteggiamento nei confronti del tradimento, perché la relazione con l'amante, impossibile da continuare, provoca in lui dolore e disperazione. Soffre, roso dal desiderio di continuare la sua relazione con l'amante Anna, il cui nome viene ripetuto nel ritornello. Anche in questo pezzo, nel rapporto di coppia l'uomo afferma la propria identità solo da un punto di vista sessuale, quando, per esempio, parlando di Anna, afferma: «Ho dormito lì / fra i capelli suoi / io insieme a lei/ ero un uomo. / Quanti e quanti sì / ha gridato lei / quanti non lo sai/ ero un uomo.» La 'compagna ufficiale' sembra relegata a un ruolo subalterno. Evocata indirettamente tramite il pronome relativo con valore indefinito "chi», incarna il tradizionale modello di donna-moglie al servizio dell'uomo ("la mattina c'è chi / mi prepara il caffè)). Appare pronta a soddisfare ogni sua richiesta ("e la sera c’è chi/ non sa dirmi no»), si direbbe più per dovere coniugale che per altro, in opposizione all'amante Anna che non dice, ma ripete e grida con passione «si». La relazione con Anna viene esclusivamente presentata in termini sessuali; l'iniziativa appare presa solo dall'uomo che riceve in ogni caso una risposta affermativa ${ }^{15}$. Tuttavia, la classica immagine dell'uomo forte è controbilanciata da quella dell' «innamorato sofferente», che non teme di rivelare verbalmente a un interlocutore sconosciuto il proprio dolore e arriva persino a esplicitarlo fisicamente piangendo ${ }^{16}$. Questo comportamento era considerato ai tempi certamente poco virile, piuttosto femminile o infantile, e corrispondeva a un'immagine maschile a cui il pubblico era meno abituato. Anche il fatto di annunciare ripetutamente, quasi teatralmente, le lacrime, come pure la ripetizione in crescendo della frase «voglio Anna», evoca modalità d'espressione tipicamente infantili dinanzi a un oggetto fortemente desiderato ma impossibile da ottenere. A differenza di un bambino, però, l'uomo vive la perdita dell'oggetto di desiderio in un modo così profondo da perdere la propria sicurezza, i propri punti di riferimento, fino a constatare che «resta poco» di lui. Come sottolinea

15. E questo sia che la donna accetti con convinzione, come Anna, o che acconsenta passivamente, come la moglie.

I6. «Non hai mai visto un uomo piangere/ apri bene gli occhi sai perché tu ora lo vedrai/ apri bene gli occhi sai perché tu ora lo vedrai / se tu.../ non hai mai visto un uomo piangere guardami... / guardami/ guardami/ guardami.» 
Salvatore ${ }^{17}$, in questo «lamento d'amore», solo l'urlo finale permette al protagonista di liberarsi, di esternare una sofferenza troppo profonda, rompendo la tensione creatasi progressivamente. Ben diverse sono quindi le conseguenze del tradimento per l'uomo di Tanta voglia di lei e di Anna, in quanto, pur ritrovando in entrambe certi stereotipi dell'uomo maschilista, si rileva nella seconda canzone anche l'immagine di un uomo che non si vergogna di rivelare la propria fragilità.

Verso la fine degli anni Settanta, nei brani che cantano l'infedeltà ${ }^{18}$, insieme a vecchie immagini appaiono elementi che riflettono l'evoluzione dei costumi in atto.

In Sotto il segno dei pesci (Venditti), classificatasi al secondo posto nella hit parade del 1978 , il protagonista, insoddisfatto della sua vita, rivendica il diritto all'Amore con l'A maiuscola, senza però ricorrere a dirette evocazioni sessuali. È interessante notare la presenza di alcuni moduli espressivi sottolineati nella canzone Anna, come il dialogare con un personaggio esterno - qui, però, chiaramente identificabile nella persona amata nel passato - e la ricorrenza del verbo "volere», abbinata a «amore e unità per noi» ${ }^{19}$. L'accento viene messo piuttosto su una relazione sentimentale condivisa — ribadita dalla ripetizione «unità per noi» — in cui il protagonista non cerca di affermare la propria identità di uomo. Venditti parla alla donna amata anni prima mettendo a confronto passato e presente. Nell'alternarsi di verbi all'imperfetto e al presente traspare il fatto che le aspirazioni e i sogni del passato sono ancora vivi, ma su uno sfondo d'insoddisfazione e delusione che coinvolge altre persone, come Marisa e Giovanni. La storia d'amore rinvia a un contesto caratterizzato da lotte sociali evocate attraverso immagini di cortei di protesta («quella strada [...] e la gente che correva, e gridava insieme a noi») in un periodo 'politicamente impegnato'. In questo squarcio di vita passata, presumibilmente databile alla fine degli anni Sessanta, troviamo un uomo e una donna apparentemente impegnati tra manifestazioni e discussioni, soggetto nuovo nelle canzoni più gettonate. Nonostante questo, ritroviamo elementi nei quali ci siamo già imbattuti, che vedono la figura maschile cercare una

17. Gianfranco Salvatore, Mogol-Battisti. L'alchimia del verso cantato. Arte e linguaggio della canzone moderna, Roma, Castelvecchi, 1997, pp. 24I-242.

I8. Ricordiamo che la parola «moglie» non appare mai, ma per comodità la introduciamo per indicare "la compagna ufficiale» contrapposta alla "compagna di una sera»e all' «amante».

19. "Tutto quello che voglio, pensavo, è solamente amore, ed unità per noi; [...] tutto quello che voglio, dicevo, è solamente amore ed unità per noi; [...] E Marisa [...] tutto quel che cerca e che vuole è solamente amore ed unità per noi; [...] E Giovanni [...] tutto quel che cerca e che vuole è solamente amore ed unità per noi.» Il verbo «volere» compare pure nella domanda posta indirettamente all'amica, riguardo alla condivisione di comuni aspirazioni e rivendicazioni: «[...] noi, che meritiamo un'altra vita, più giusta e libera se vuoi; [...] noi, che meritiamo un'altra vita, violenta e tenera se vuoi.» 
nuova compagna a fianco di quella 'ufficiale', come appare nelle osservazioni della ragazza: "[...] che ti manca, una casa tu ce l'hai, hai una donna, una famiglia, che ti tira fuori dai guai». Se, da un lato, queste parole ricordano quelle dell'interlocutore di Battisti, che cerca di riportare razionalmente alla realtà quotidiana l'amico, dall'altro ci mostrano l'immagine di una donna critica e non più remissiva nei confronti dell'uomo. S'instaura, infatti, un vero dialogo e confronto tra i due (le "nostre discussioni») — caratterizzato da domande («mi chiedevi»), riflessioni e ripensamenti ("pensavo, dicevo») - che rinvia a un rapporto di coppia paritario. Per l'uomo l'immagine di questa nuova donna convive, però, con quella della compagna ufficiale, che fa parte dei 'beni ormai acquisiti' su cui basare la propria sicurezza, insieme alla casa e alla famiglia. In questo pezzo, però, l'infedeltà è giustificata dalla rivendicazione del diritto di vivere l'amore al di fuori delle convenzioni imposte, nella prospettiva di una nuova società in cui poter avere «un'altra vita più giusta e libera/ [...] violenta e tenera/ stretti in una libera sorte». Se queste affermazioni riflettono soggetti di dibattito frequenti in quegli anni, nello stesso tempo rinviano a un modo di pensare tipicamente giovanile, caratterizzato dall'entusiasmo e dalla fiducia di poter cambiare il mondo. Lo stesso protagonista, nella seconda parte della canzone, constata che quel periodo è ormai passato portando con sé sogni e progetti. Ormai i personaggi sono degli adulti che lavorano: Marisa è insegnante e Giovanni lavora in una radio. Quello che resta è la sensazione di un'insoddisfazione generale e generazionale non nuova per questi «figli di una vecchia canzone».

\section{Che cosa rappresenta la donna per l'uomo?}

Nelle canzoni di questi anni, la donna è vista frequentemente dall'uomo come uno strumento d'affermazione della propria virilità e come possibilità d'evasione dalla routine di coppia, dove la moglie è un punto di riferimento fisso verso il quale tornare, chiedendo a volte perdono - altro tema frequente.

In Ti amo (Bigazzi-Tozzi) ${ }^{20}$, al secondo posto nella hit parade del I977, l'interprete ci presenta due tipi di relazioni con due donne diverse alle quali rivolge la stessa frase, «ti amo». Nel primo caso, però, questo senti-

20. Questa canzone deve in parte il suo grande successo anche internazionale a una melodia molto orecchiabile, caratterizzata dalla semplicità della sua struttura armonica basata su un "giro di do» e dalla ripetizione del suo titolo. Le parole "Ti amo" sono scandite per ben diciassette volte dal cantante, mentre il coro le ripete in sottofondo. 
mento finisce per esser anche odio, nel momento in cui l'uomo decide di porre fine alla relazione per ritornare dalla 'moglie'. Il modo di porsi nei riguardi delle due donne riprende vecchi stereotipi, come la virilità affermata dal punto di vista sessuale, anche se il modo di espressione ricorre a un vocabolario più crudo: "nel letto comando io", «'amore che a letto si fa». Con la prima donna, l'uomo gioca il ruolo del macho che domina la compagna e decide da solo d'interrompere una relazione ${ }^{21}$, probabilmente a carattere prevalentemente sessuale, ritenuta effimera, come ci suggerisce l'immagine della farfalla: «è una farfalla l'amore che a letto si fa». Tuttavia, egli non si dimostra insensibile e non teme di mostrarsi qual è, anche nelle sue debolezze - «tremo davanti al tuo seno» - e in fondo, chiedendo all'amante "rendimi l'altra metà», ci rivela di averle dato solo a metà il suo cuore, sottinitendendo che l'altra era riservata alla seconda donna.

Il seguito del pezzo è invece dedicato a quella che potremmo chiamare 'moglie', verso la quale ritorna il protagonista con un certo imbarazzo, dovuto alle spiegazioni che dovrà darle a proposito dell'infedeltà compiuta ( oggi ritorno da lei [...] su coraggio / io ti amo e ti chiedo perdono»). Per lui, questa seconda donna riveste il ruolo della moglie-madre rassicurante che, in cambio di un amore dichiarato ripetutamente, deve esser pronta a dare tutto quanto le si chiede su un tono imperativo: «apri la porta, [...] dammi il tuo vino leggero / che hai fatto quando non c'ero / e le lenzuola di lino/ dammi il sonno di un bambino», «fammi abbracciare una donna che stira/ cantando». Il protagonista si comporta ancora da macho, pur evocando atteggiamenti un po' infantili: ripete, infatti, alla madre-moglie che l'ama, la vuole abbracciare mentre essa assume 'serenamente' il fatto di stirare, scherza con lei per farsi perdonare della 'marachella' commessa; e poiché, per lui, questa donna ha un ruolo subalterno, deve accettare col sorriso anche la richiesta di fare l'amore superando il suo evidente rancore - «e poi fatti prendere in giro/ prima di fare l'amore/ vesti la rabbia di pace». Se il protagonista ammette la propria debolezza definendosi «un guerriero di carta igienica», ribadisce nello stesso tempo il suo ruolo dominante nella coppia.

\section{Si può parlare di tradimento femminile nelle canzoni?}

Se il tradimento femminile appare nelle canzoni, le reazioni degli uomini non sono passive come quelle delle loro compagne tradite. Spesso, offesi

2I. A dire la verità, il protagonista si affida al caso, sperando che la moneta lanciata in aria ricada sul lato testa, scelto per porre fine a quella relazione. 
nel proprio orgoglio, gli uomini accusano la traditrice a cui possono, però, chiedere di tornare.

Nella Canzone del sole (Battisti-Mogol), arrivata al quinto posto nella hit parade del 1972, il protagonista rimprovera la ragazzina dalle trecce bionde, diventata "una donna ormai», con sottinteso giudizio negativo: «Dove sei stata cos'hai fatto mai?» In realtà, qui non possiamo parlare d'infedeltà come nelle canzoni precedenti, perché questa canzone racconta l'incontro, dopo un certo lasso di tempo, tra due persone che si erano amate da fanciulli. Tutto il brano è costruito sul ricordo del passato e la realtà presente di individui ormai adulti. Il personaggio maschile non riesce a riconoscere nella donna davanti a sé la ragazzina con cui aveva condiviso un'iniziazione all'amore («e la cantina buia dove noi / respiravamo piano»). La maturazione della fanciulla è ridotta prettamente all'aspetto sessuale, al fatto che essa abbia avuto esperienze con altri uomini, cosa probabilmente rifiutata al protagonista («e le tue corse, e l'eco dei tuoi no»). Il protagonista non tiene assolutamente conto del fatto che, nel diventare adulto, anche lui avrà avuto delle esperienze amorose, cosa comunemente ammessa e valorizzante per un uomo, ma condannata per una donna in quanto considerata come una perdita d'innocenza. I ruoli tradizionali uomo-donna vengono dunque rovesciati, perché qui il personaggio femminile si definisce in quanto donna, affermando la propria sessualità («sono una donna ormai») e senza sentire la necessità di doversi giustificare («Ma quante braccia ti hanno stretto, tu lo sai, / per diventar quel che sei, / che importa tanto tu non me lo dirai, purtroppo»), atteggiamento usato prevalentemente dai personaggi maschili delle canzoni. L'uomo non è preparato a questo nuovo tipo di donna e, avendo perso il suo ruolo tradizionale di seduttore, vacilla e ha paura («mi stai facendo paura», «non so più chi sei, / mi fai paura oramai»). S'imbatte ora in una donna emancipata, dal «sorriso sicuro", che osa prendere pure l'iniziativa: «e d'improvviso quel silenzio fra noi / e quel tuo sguardo strano [...] e poi, / oh no, ferma, ti prego, la mano». Segno di una certa evoluzione, anche se lenta e sofferta, dell'atteggiamento maschile nei confronti dell'emancipazione femminile è la scoperta, dopo un primo momento di sgomento, che il sentimento dell'amore non è alterato dalla «liberazione sessuale», concetto espresso attraverso la metafora poetica dell'ultima strofa ${ }^{22}$.

22. «Il sole quando sorge, sorge piano e poi/ la luce si diffonde tutto intorno a noi/ le ombre ed i fantasmi della notte sono alberi/ e cespugli ancora in fiore/ sono gli occhi di una donna/ ancora piena d'amore.» Ci pare utile accennare brevemente alla canzone del lato B del 45 giri I giardini di marzo (Mogol-Battisti, terza della hit parade 1972), Comunque bella, che ci parla di un doppio tradimento, da parte maschile e femminile, e delle 
Nelle canzoni più vendute degli anni Settanta è raro trovare un uomo che accetta, seppur con difficoltà, questo nuovo modello di donna. Fra queste vorrei ricordare La Fata di Edoardo Bennato, che non è stata presentata in versione single ma che si trova nell'LP Burattino senza fili, l'album più venduto del 1977. Le canzoni di questo 33 giri ripropongono i personaggi della storia di Pinocchio in chiave metaforica. Per trasformarsi in bambino, il burattino Pinocchio deve abbandonare certi modelli veicolati dalla cultura dominante e diffusi nella società. Il personaggio di questo pezzo è la Fata Turchina, pretesto per una denuncia in chiave femminista dell'immagine della donna ancora presente negli anni Settanta in Italia. Lautore denuncia il fatto che la donna continui a essere rinchiusa nell'immagine di «sorella e madre e sposa / [...] regina o fata», e che non possa "pretendere di più». Solo rimanendo all'interno di questi modelli codificati la donna suscita ammirazione e amore; se invece vuole spingersi («volare») al di là dello stereotipo di angelo del focolare, alla ricerca di un amore diverso e profondo, viene condannata, rischiando pure di essere dominata («c'è chi $[. .$.$] / ti fa schiava») o ridotta a puro oggetto di piacere ("c'è chi ti$ espone anche in vetrina»). Ricorrendo a temi, immagini e termini tipici dei dibattiti femministi dei tempi ${ }^{23}$, su una musica delicata «dal sapore intimistico» ${ }^{24}$, Bennato confessa che la donna non può realizzare i propri desideri in una società in cui deve adeguarsi e piegarsi ai desideri maschili, ed essa risulta pertanto discriminata.

In Anche per te (Mogol-Battisti) del I97I, lato B del 45 giri La canzone del sole, appaiono tre figure insolite di donna nei testi delle canzoni: una religiosa, una prostituta e una ragazza-madre di cui il protagonista sottolinea la vita di sacrifici e solitudine. All'inizio degli anni Settanta il fatto di avere un figlio al di fuori di un legame di coppia riconosciuta è considerato "un errore che costa tanto", di cui pentirsi, un ostacolo nel costruire nuove relazioni serie — « «tu] che tremi nel guardare un uomo e vivi di rimpianto».

Rimanere incinta al di fuori del matrimonio è visto negativamente anche alla fine degli anni Settanta. Come ricorda Venditti in Sara, lato B di Sotto il segno dei pesci (1978), canzone che ci racconta la storia di due studenti ${ }^{25}$, lo sguardo della società italiana a questo proposito rimane

diverse reazioni davanti al fatto compiuto. In questa canzone, l'infedeltà della donna è presentata con termini più espliciti quanto al lato sessuale, ed è vissuta dall'uomo talmente male che la relazione finisce.

23. Da notare l'immagine della strega che evoca lo slogan «Le streghe son tornate».

24. Cfr. Jean Guichard, Il Pinocchio cantato: canzoni per un burattino, in Pinocchio esportazione: il burattino di Collodi nella critica straniera, a cura di Giorgio Cusatelli, Roma, Armando, 2002, p. 135.

25. Sara fa le superiori e lui l'università. 
piuttosto critico: «Sara, tu va dritta non ti devi vergognare/ le tue amiche dai retta a me lasciale tutte parlare». Lo sfondo della storia non appare però triste come nella canzone di Battisti. Il cantautore mette l'accento sul fatto che il futuro bambino non è il frutto di una relazione solo sessuale («è stato solo amore»), come a dire che la ragazza non dev'essere moralmente condannata per quanto successo ${ }^{26}$. È interessante osservare l'atteggiamento egoista del protagonista maschile che non assume alcuna responsabilità davanti all'incidente', ma lascia la compagna sola ad affrontare la gravidanza e la futura nascita del figlio, rassicurandola ipocritamente: "Tu non sei più sola, il tuo amore gli basterà / il tuo bambino, se ci credi nascerà». Il figlio è una storia che riguarda solo lei, poiché lui ha altri progetti per la vita — «mi devo laureare» - progetti dai quali la ragazza risulta esclusa. In effetti, il matrimonio più o meno 'riparatore' resta solo un'eventualità: "forse un giorno ti sposerò / magari in chiesa, dove tua madre sta aspettando per poter pianger un po'». Il matrimonio religioso resta fondamentale, soprattutto per i genitori, in quanto viene considerato come il coronamento e la sacralizzazione dell'unione tra due persone.

Possiamo quindi dire che nei pezzi eseguiti da interpreti maschili la vita sessuale al di fuori del matrimonio o di un legame stabile risulta comportare per la donna dei pericoli sia a livello 'psicologico', in quanto essa si trova esposta a una condanna d'ordine morale, sia a livello fisico, per il rischio di ritrovarsi incinta. Su quest'ultimo punto, non dobbiamo dimenticare che gli anni Settanta sono stati caratterizzati da un largo dibattito pubblico sulla sessualità e la contraccezione, temi considerati tabù nei decenni precedenti ${ }^{27}$.

\section{Che cosa cantano le donne?}

Le critiche sui comportamenti sessuali toccano esclusivamente le donne e restano particolarmente forti nel sud, come possiamo constatare nelle canzoni Sono una donna, non sono una santa (Sciorilli-Testa) cantata da

26. Come precisa Berselli, nelle canzoni solo «l'innamoramento romantico è il tramite necessario per far saltare le norme che presidiano l'illibatezza» (Edmondo Berselli, Canzoni. Storie dell'Italia leggera, Bologna, Il Mulino, 2007, p. 33).

27. Per rievocare l'atmosfera di quegli anni, ricordiamo che l'abrogazione dell'articolo del codice penale che vietava la propaganda e l'utilizzo di qualsiasi mezzo contraccettivo risale al I97I ed è in gran parte dovuta all'impegno dell'AIED (Associazione Italiana per l'Educazione Demografica). In Italia, la pillola era già disponibile in farmacia dal 1965 , ma era ufficialmente prescritta per curare problemi mestruali ed era soprattutto riservata a donne sposate. Nel 1976, il ministro della Sanità abrogò le norme che vietavano la vendita della pillola anticoncezionale autorizzando l'indicazione "contraccettivo" sul foglietto illustrativo. 
Rosanna Fratello, arrivata al ventiduesimo posto della hit parade del I972, e Ragazza del Sud (Rosangela Scalabrino, alias Gilda) di Gilda, vincitrice del Festival di Sanremo del 1975. Le due cantanti si presentano come un baluardo di valori a cui una gran parte degli italiani e delle italiane restava sempre fedele. Non deve quindi apparire così sorprendente il fatto che le parole di Ragazza del Sud siano state scritte da una donna (cosa rara per le canzoni degli anni Settanta), piemontese e studentessa di medicina ${ }^{28}$, in una società in piena evoluzione, dove ideali conservatori e progressisti si affrontavano quotidianamente.

In questi due pezzi, la donna, rispettando la tradizione e la morale cattolica $^{29}$, deve arrivare vergine al matrimonio perché, diversamente, disonorerebbe ${ }^{30}$ la famiglia, come sottolinea Rosanna Fratello: «Non sono sola, ho quattro fratelli / non scordare che ho quattro fratelli / dove sei stata, la gente ti guarda/ resta in casa, la gente ti guarda». La verginità è evocata pudicamente con la parola «amore» — «fra tre mesi, te lo prometto/ che il mio amore tu l'avrai / [...] fra tre mesi saremo in maggio/ e il mio amore ti darò / Gesù mio dammi coraggio / di resistere e dirgli di no» - e la sessualità appare come un peccato, un male da evitare invocando addirittura l'aiuto di Gesù per non cedere alla tentazione di quel diavolo di fidanzato. Ai tempi si parlava della "prova d'amore» chiesta dagli uomini alle loro fidanzate. Ma spesso succedeva che, una volta ottenuto il pegno d'amore, gli uomini rinfacciassero alla donna la mancanza di serietà e l'abbandonassero.

Gilda esalta l'immagine tradizionale della donna remissiva e illibata contrapponendole quella della donna che «il primo bacio» l'ha già avuto. $\mathrm{Ne}$ risulta che, per la cantautrice, una certa emancipazione sessuale e modernità corrispondano all'abbandono di un mondo idilliaco e puro (non lontano da quello adolescenziale della Canzone del sole), dove i sogni sono ancora possibili. Il consiglio rivolto alle giovani italiane è semplice e convincente in quanto trasmesso da una donna più esperta che conosce la vita:

28. Questa canzone, eseguita dalla stessa autrice, pur vincendo a Sanremo non diventò un successo commerciale, segno della sensibilità diversa del vasto pubblico rispetto alla giuria del festival.

29. Cfr. la prima strofa di Ragazza del Sud in cui la cantante esalta il modello d'educazione trasmesso alle ragazze da secoli: «Ragazza che ti affretti/ perché suona la messa/ cammini a testa bassa/ [...] ti hanno insegnato a creder / a vivere aspettando... lui $[\ldots]$ / sarà il tuo primo amore».

30. Ricordiamo che in Italia le disposizioni relative al delitto d'onore sono state abrogate soltanto nel I98I (legge n. 442). Prima di questa data, l'articolo 587 del Codice penale prevedeva che fosse ridotta la pena per chi uccidesse la moglie (o il marito, nel caso in cui la tradita fosse stata la donna), la figlia o la sorella fedifraghe al fine di difendere «l'onor suo o della famiglia». La circostanza prevista richiedeva che vi fosse uno stato d'ira. Il reato era considerato «un'illegittima relazione carnale» che coinvolgeva una delle donne della famiglia. Si dava per acquisito che questa relazione illegittima costituisse offesa all'onore. Anche l'altro protagonista della relazione poteva dunque essere ucciso contro egual sanzione. 
non bisogna aspirare a modelli di vita diversi, perché questi comportano solo delusioni. L'eventuale aspirazione a un'emancipazione della Ragazza del Sud, che guarda "ammmirata i vestiti» ${ }^{31}$ dell'altra donna, è chiaramente frenata, suggerendo di continuare a perpetuare modelli tramandati dalle generazioni passate, come rimanere a «ricamare il nome sul lenzuolo» aspettando il suo Lui.

Meglio allora per le ragazze non incorrere in rischi e seguire i saggi consigli di Finché la barca va (Pace-Panzeri-Pilat), diciasettesima canzone italiana più venduta nel 1970, dove, in veste di sorella maggiore e al ritmo di un liscio, Orietta Berti ricorda che castità e fedeltà sono valori essenziali per una donna, se non vuol rischiare di ritrovarsi da sola ${ }^{32}$. La cantante, ricorrendo alla fine della canzone per ben tre volte alla metafora del «cancello", invita dunque le ragazze a seguire il suo comportamento per avere una vita tranquilla, frenando desideri e curiosità, evitando avventure sentimentali: «Stasera mi è suonato il campanello, / è strano io l'amore ce l'ho già, / vorrei aprire in fretta il mio cancello, / mi fa morire la curiosità./ Ma il grillo disse un giorno alla formica: / "Il pane per l'inverno tu ce l'hai"; / vorrei aprire in fretta il mio cancello, / ma quel cancello io non l'apro mai!»

In effetti, nei sondaggi degli anni Settanta il matrimonio occupa sempre - e di gran lunga - il primo posto nella classifica delle aspirazioni delle donne. Rimanere 'zittelle' era mal visto e mal vissuto, al contrario di quello che succedeva per i cosiddetti 'scapoloni' impenitenti.

Notiamo che nelle classifiche dei primi anni Settanta convivono canzoni che propongono schemi vecchi e nuovi. Tra queste ultime, ve ne sono, infatti, alcune che riflettono il clima di ridiscussione del ruolo della donna nella società e nel privato, offrendo immagini di donne meno sottomesse e più libere. Soffermiamoci su qualche canzone eseguita dalla regina della canzone italiana, Mina.

Nel I970, si ascoltava, accanto a La barca va, Insieme (Mogol-Battisti), arrivata seconda nella hit parade dell'anno. In questo pezzo la protagonista è pronta a vivere una passione profonda senza porsi domande sui suoi tempi e modalità — «non ti chiedo sai quanto resterai». Non c’è più bisogno di un fidanzato, come per Rosanna Fratello o Orietta Berti, per realizzare i propri sogni: anzi, la protagonista si abbandona perdutamente

3I. A questo proposito rinviamo alla esecuzione di questa canzone a Sanremo dove la cantante è accompagnata da un coro femminile che indossa costumi regionali, il che contribuisce a dar un'immagine anacronistica della visione dell'Italia del sud rinforzandone certi stereotipi diffusi soprattutto nel nord Italia: <www.youtube. $\mathrm{com} /$ watch?v=cKIvXoqXgww> (data di ultima consultazione 8 dicembre 20I2).

32. "[...] mia sorella che aveva un fidanzato di Cantù, / voleva averne uno anche in Cina e il fidanzato adesso non l'ha più." 
alla fatalità della passione per un uomo che non conosce neppure bene, e che ha "cancellato con un gesto i sogni» suoi. Come illustra bene il titolo della canzone, ciò che più conta è il fatto di condividere dei momenti intensi, fosse "anche solo per un giorno». Mina fa parte di quelle cantanti che, negli anni Settanta, si emancipano dal modello dell'interprete languida o distaccata dal testo cantato. Il pubblico, vedendo una sorta di immedesimazione tra la cantante e il testo cantato, aveva l'impressione che queste interpreti femminili esprimessero parte del loro vissuto. Mina, ormai riconosciuta come grande artista, incarna il modello di una donna nuova, dalle scelte coraggiose e persino anticonvenzionali. Pensiamo, ad esempio, alla sua nota relazione con l'attore Corrado Pani ancora sposato, seppur separato, e al grande scandalo provocato nel 1963 dalla nascita di un loro figlio, fatto che provocò il suo allontanamento dalla RAI per due anni. Ne risulta l'immagine di una donna più matura e meno passiva che, in Grande grande grande (Testa-Renis), canzone giunta al primo posto nella hit parade del 1972, si pone allo stesso livello del compagno cercando il confronto con lui, come si può vedere sin dal verso iniziale: «con te dovrò combattere / [...] la vuoi sempre vinta tu». E indirettamente emerge pure la figura di un uomo nuovo, non più 'dominatore' ma aperto al dialogo, che vuole al suo fianco una compagna con cui condividere una relazione ricca e completa. Non vi sono allusioni sessuali: tutto è incentrato sul 'rapporto costruttivo' di una coppia in cui la donna si crea un ruolo nuovo che, pur comportando delle difficoltà, la fa sentire realizzata: «in guerra tutti i giorni sono viva». Si tratta di una donna che sa tener testa al compagno - riconosciuto egoista e prepotente - fino a trasformarlo e ammirarlo, dimenticando i momenti difficili: «ma c’è di buono che al momento giusto/ tu sai diventare un altro, / in un attimo tu/ sei grande, grande, grande, le mie pene/ non me le ricordo più». La canzone sottolinea le diverse mentalità di questa donna e del suo compagno che si allontanano dai comportamenti tradizionalmente diffusi nelle coppie del tempo: «Io vedo tutte quante le mie amiche/ son tranquille più di me/ non devono discutere ogni cosa/ come tu fai fare a me, / [...] dicon sempre di sì / non hanno mai problemi e son convinte/ che la vita è tutta lì. / Invece no, invece no / la vita è quella che tu dai a me / in guerra tutti i giorni sono viva/ sono come piace a te.» Pur essendo in coppia, non compare mai il pronome «noi», ognuno mantiene distinta la propria identità, come vediamo dal largo uso dei pronomi personali «io», «tu», «me», «te».

Mina aveva già rotto con gli schemi dell'immagine della donna 'tradizionale', sia per i contenuti delle canzoni sia per l'interpretazione che ne dà. Ma si spinge oltre con L'importante è finire (Anelli-Malgioglio) 
— classificatasi al secondo posto nella hit parade del I975 - in cui la protagonista canta il piacere di un amplesso. Alcuni verbi il cui senso era troppo esplicito furono censurati. Ciononostante, la canzone ebbe un successo straordinario, legato all'interpretazione molto allusiva e sensuale di Mina. Nelle sue prestazioni più famose ${ }^{33}$ scopriamo il potere della cantante che, pur ripresa solo in primo piano, grazie agli sguardi e ai movimenti delle mani riesce a evocare esplicitamente quanto detto nel testo della canzone, suggerendo un'atmosfera peccaminosa. Per la prima volta in una canzone leggera una donna osa lodare il «talento da grande [...]/ nel fare l'amore» del suo lui, in un periodo in cui il tema dell'orgasmo femminile o maschile comincia a essere affrontato pubblicamente, ma ancor timidamente, eccezion fatta per i gruppi di parola femministi. La canzone risultava tanto più provocatoria in quanto la protagonista era una donna che, sebbene insoddisfatta da una relazione a cui vorrebbe porre fine per mancanza di sentimenti, pur esitante e dubbiosa ${ }^{34}$, soccombe con piacere alle ripetute avances dell'uomo. Le parole, crude e precise, tendono a metter in luce il lato 'animale' dell'uomo: «ha il respiro un po' caldo / [...] ha il volto sconvolto ${ }^{35}$ / [...] violento il respiro». Tuttavia, se il personaggio maschile riveste solo il ruolo del maschio seduttore, che alla dichiarazione d'amore della compagna sa solo rispondere riproponendo un rapporto sessuale, non abbiamo, però, la sensazione che questa donna subisca veramente quanto succede. La sensualità della gestualità di Mina, le parole «e poi...» ripetute quattro volte fino ad abbassare gli occhi, lasciando immaginare il seguito come pure il tardare a pronunciare l'ultima parola del verso «l'importante è... finire» — sulla quale ricade evidentemente l'attenzione dell'ascoltatore - evocano maliziosamente un piacere a cui è difficile rinunciare.

Rileviamo che alla fine degli anni Settanta e inizio anni Ottanta le cantanti riescono persino a evocare il tema dell'autoerotismo in canzoni di cui sono a volte anche le autrici, come Gianna Nannini con America (Nannini-Paoluzzi) o Fiorella Mannoia con Caffe nero bollente (CoggioDavini-Cavallo-De Cola), canzone presentata addirittura al Festival di Sanremo del $198 \mathrm{I}^{36}$.

33. Cfr. <www.youtube.com/watch? $=$ RCs7XGlAj2Q $>$ (data di ultima consultazione 8 dicembre 20I2).

34. "Ho deciso lo mollo, / ma non so se poi farlo / [...] questa è l'ultima volta che lo lascio morire».

35. Curioso notare il ricorso allo stesso aggettivo, "sconvolto", in Comunque bella di Battisti, attribuito allo sguardo della donna uscita da una notte d'amore. Il senso di questo aggettivo rinvia, in entrambi i pezzi, al travolgimento fisico di una relazione solamente sessuale.

36. America uscì come single estratto dall'album California (1979), che figurò per sei mesi tra i dieci album italiani più venduti nella classifica del 1980. America ha fatto conoscere al largo pubblico questa cantante dagli atteggiamenti anticonvenzionali, già nota per le sue prese di posizione femministe. C'è comunque da notare 


\section{Temi nuovi}

Fra i temi nuovi che compaiono nelle canzoni degli anni Settanta, ci limitiamo a segnalare l'aborto e il 'triangolo', trattati in canzoni che hanno suscitato interesse e successo ${ }^{37}$.

\section{L'aborto}

In alcuni testi delle canzoni fin qui analizzate si delineano nuove figure di uomini e donne che corrispondono meglio all'evoluzione dei costumi in atto nella società degli anni Settanta: l'incrinarsi dell'immagine del macho (o meglio delle modalità di esternare i propri stati d'animo), la presa di coscienza femminile del diritto a una sessualità da poter vivere con soddisfazione anche al di fuori della coppia legalizzata, la ricerca di ruoli paritari nella relazione a due. Dobbiamo notare anche una certa evoluzione della lingua, che si fa più vicina al parlato sia a livello lessicale che sintattico ${ }^{38}$, e l'introduzione di specchietti della vita quotidiana, in particolare nelle canzoni di Mogol e Battisti. Ciononostante, la scena pubblica e i grandi dibattiti che hanno animato quegli anni sono per lo più ignorati o evocati superficialmente. In effetti, intuiamo più che vedere le tendenze progressiste dei costumi, ma queste si rivelano solo in rappresentazioni di storie individuali.

che siamo ormai alla fine degli anni Settanta e, soprattutto, nell'ambito di una musica rock, dove i testi sono più trasgressivi rispetto a quelli proposti dalle canzoni della musica melodica, elementi che spiegano la mancata censura della canzone. Interpreti maschili avevano già evocato il soggetto della masturbazione. Fra le canzoni più conosciute di quel periodo, ricordiamo quelle dei cantautori Lucio Dalla, Disperato Erotico Stomp (1977), e Francesco Guccini, L'avvelenata (1976).

37. Fra i temi trasgressivi troviamo anche l'omosessualità e il transessualismo. Tuttavia, nei primi dieci successi delle hit parade del periodo studiato questi temi non appaiono mai, a riprova del fatto che, malgrado una certa evoluzione delle mentalità, essi restino ancora dei tabù. A titolo d'esempio, ricordiamo le canzoni Andrea di Fabrizio De André (1978), Lei di Gianna Nannini (1979), Giulia di Venditti (1978), Tu che sei mio fratello di Renato Zero (1974), Pierre dei Pooh (1976). Per un censimento e analisi di canzoni italiane sui temi dell'omosessualità, lesbismo, bisessualità, transessualismo, cfr. <www.giovannidallorto.com/canzoni/index.html>, e Giovanni Dall'Orto, Stefano Casi, Il gay canzonato - L'omosessualità nelle canzoni italiane, in "Babilonia», 39, settembre 1986, pp. 48-52, <www.giovannidallorto.com/canzoni/canzonir.html> (data di ultima consultazione 8 dicembre 20I2).

38. Per quanto riguarda l'evoluzione della lingua nella canzone italiana, ricordiamo, fra i tanti studi: Giuseppe Antonelli, Ma cosa vuoi che sia una canzone, cit.; Gabriella Cartago, La lingua della canzone, in La lingua italiana e i mass media, a cura di Ilaria Bonomi, Andrea Masini, Silvia Morgana, Roma, Carocci, 2002, pp. 199222; Michele Cortellazzo, La lingua della canzone d'autore nella storia dell'italiano contemporaneo, in Tradurre la canzone d'autore, Atti del Convegno, Milano, Università Bocconi, 29 settembre 1997, a cura di Giuliana Garzone, Leandro Schena, Bologna, CLUEB, 2000, pp. 2I-34; Lorenzo Coveri, Per una storia linguistica della canzone italiana, in Parole in musica. Lingua e poesia nella canzone d'autore italiana, a cura di Lorenzo Coveri, Novara, Interlinea, 1996, pp. 13-24; Anna Infanti, La lingua della canzone, in "Quaderni dell'osservatorio linguistico", I, 2002, pp. I33-I60. 
Un tema come l'aborto, per esempio, è al centro di canzoni impegnate di cantautori come Francesco Guccini - Piccola storia ignobileo Gianna Nannini - Morta per autoprocurato aborto - che toccavano però un pubblico ristretto e non circolavano di certo sulle radio o reti televisive pubbliche. Queste due canzoni, che denunciano la piaga degli aborti clandestini, escono nel $1976^{39}$, in pieno clima di feroci dibattiti che investiva l'intero sistema politico, sanitario e morale italiano. I due cantautori si schierano a favore dell'interruzione volontaria della gravidanza proponendo le tristi storie di due donne che hanno vissuto sulla propria pelle la sofferta esperienza dell'aborto, che termina per una con la morte ${ }^{40}$. E sorprendente che, sempre nel 1976, l'aborto sia evocato nella canzone che arriva al terzo posto al festival di Sanremo - Volo AZ 504 (CutugnoPallavicini) cantata dagli Albatros - a sottolineare il fatto che l'aborto era comunque al centro dei dibattiti di tutti, a due anni dalla sua legalizzazione. Ciò che colpisce di più, però, è la 'disinvoltura' con cui viene trattato questo argomento, ben lungi dalle denunce espresse nelle due canzoni precedentemente menzionate ${ }^{41}$. Dalle parole rivolte alla donna da parte del protagonista maschile — «Potevo lasciarti avere il bambino ma... / ti rendi conto, cosa sarebbe successo? / Però forse sarebbe stato meglio... / almeno non saresti andata via» - sembra appurato, tanto da poterlo cantare, che nell'Italia del 1976 l'aborto — benché clandestino — fosse un'eventualità normalmente presa in considerazione per risolvere una gravidanza inaspettata, sia praticato in paesi stranieri dove era legalizzato, sia clandestinamente in Italia. Nessun cenno alle sofferenze legate a una scelta

39. Solo il complesso delle Orme aveva già osato trattare questo soggetto delicato, ma con parole più velate, con La fabbricante d'angeli del 1974 .

40. Risuonano particolarmente dure le parole dei due cantanti che denunciano modalità e tragiche conseguenze fisiche e psicologiche di aborti effettuati, in quanto vietati, al di fuori d'ogni assistenza medica. Guccini, rivolgendosi alla protagonista, sottolinea: «E così ti sei trovata come a un tavolo di marmo/ desiderando quasi di morire, / presa come un animale macellato stavi urlando, / ma quasi l'urlo non sapeva uscire/ e così ti sei trovata fra paure e fra rimorsi davvero/ sola fra le mani altrui». La Nannini mette l'accento sul fatto che alla donna debba esser riconosciuto il diritto di prendere la decisione: «Due coltelli i testimoni di un rito che non ha padroni / un rito l'unico rimedio a libertà negate a volontà spezzate / in mezzo al sangue lei per terra vinceva la sua guerra.»

4I. D'altra parte, in quegli anni, non poteva essere diversamente per una canzone presentata a un festival con grande audimat nazionale e anche internazionale. Dobbiamo ricordare che questa canzone era arrivata a pari merito con Gli occhi verdi di tua madre (Avogadro-Giacobbe-Pace) interpretata da Sandro Giacobbe, canzone che sfidava anch'essa le leggi della morale. Infatti l'interprete racconta di come si fosse innamorato della madre della sua fidanzata a causa della somiglianza delle due donne. Se non è chiaro fino a che punto si fosse spinta la relazione, è però evidente che qualcosa d'imbarazzante era successo, come ci lasciano intuire le reazioni dell'uomo all'arrivo inaspettato della fidanzata: «i tuoi passi all'improvviso / e un tuffo al cuore immenso / ... se ci penso... / [...] tu continui a non parlare/ hai l'aria di un cigno che muore.» Malgrado i contenuti discutibili, Gli occhi verdi di tua madre e Volo $A Z$ s04 riescono a riscuotere un discreto successo commerciale: la prima sarà la sedicesima canzone italiana più venduta dell'anno, la seconda occuperà il ventiduesimo posto della hit parade del 1976. 
difficile per la donna, sulla quale l'uomo non sembra aver esitato. Se c'è un ripensamento da parte di quest'ultimo, esso è dovuto solamente all'egoistico desiderio di continuare una relazione a cui la protagonista, Sandra, ha deciso di porre fine partendo verso una meta sconosciuta.

\section{Al di là della coppia}

Vediamo ora un altro tema tabù: il 'triangolo', soggetto principale di Pensiero stupendo (Fossati-Prudente) cantata da Patty Pravo, e di Il triangolo no (Zero) di Renato Zero, canzoni classificate rispettivamente in sesta e settima posizione nella hit parade del 1978. Non si tratta del tradizionale triangolo in cui una persona ne ama segretamente un'altra pur essendo già in coppia; qui, vengono descritti chiaramente rapporti sessuali a tre, vissuti in un primo tempo con esitazione (Pensiero stupendo: «Vorrei/ non so"; Il triangolo no: "Il triangolo no, non l'avevo considerato, / d'accordo ci proverò») e apprezzati alla fine (Il triangolo no: «[...] ma il triangolo io lo rifarei...»).

La censura si era fatta meno rigida verso la fine degli anni Settanta, e i due cantanti figuravano tra i più provocatori per atteggiamenti e scelte di vita. Zero ci propone il 'triangolo maschile' (due uomini e una donna) in modo ironico ${ }^{42}$. Si tratta, infatti, di un uomo attratto da una donna a cui lascia l'iniziativa di contattarlo quando lei vuole, immaginandosi una avventura sessuale promettente, evocata dai gridolini allusivi «woooh!, $\mathrm{mm} \mathrm{mm} \mathrm{mm}$. Nonostante la prima reazione di sorpresa e sgomento, egli cede progressivamente alla curiosità superando le perplessità e cercando di adattarsi, seppur in modo un po' goffo, alla situazione: "ora spiegami dai, l'atteggiamento che dovrò adottare.../ woooh! mentre io rischierei di trovarmi al buio, / tra le braccia lui... $\mathrm{mmm} \mathrm{mmm} \mathrm{mmm}$... non è il mio tipo!»

Patty Pravo - dall'aspetto vagamente androgino e dotata di una sensualità ambigua - offre un altro tipo d'interpretazione, molto sensuale e ammiccante ${ }^{43}$, del suo 'triangolo femminile' (due donne e un uomo): l'accento è messo piuttosto sull'aspetto voluttoso del rapporto, suggerito da un gioco di sguardi e di movimenti sinuosi del corpo. Dal punto di vista dell'emancipazione femminile, è interessante notare in entrambe le

42. Cfr. l'esibizione stravangante e provocatoria in: <www.youtube.com/watch? $v=z w p j B x D z q r o>$ (data di ultima consultazione 8 dicembre 20I2).

43. Cfr. <http://www.youtube.com/watch?v=HMZaswflEIs\&feature=related $>$ (data di ultima consultazione 8 dicembre 20I2), mix di diverse esibizioni televisive. 
canzoni il ruolo attivo della donna che desidera aprirsi a nuove esperienze. Infatti, nel Triangolo no è lei che va da lui portandosi un amico, spiazzando Zero che le chiede ripetutamente: "Lui chi è?» Il protagonista si aspettava «un rapporto un po' più normale», anche se la donna aveva accennato a «una serata strana». Nella canzone di Patty Pravo già l'idea dell'eventualità — che poi si concretizzerà — del rapporto a tre implica qualcosa di eccitante, perché si tratta di una relazione trasgressiva, nello stesso tempo intrigante e invitante come un frutto proibito. Nella sensibilità e immaginario femminile tutto questo è perciò sentito come un "pensiero stupendo / [che] nasce un poco strisciando». La protagonista toglie ogni dubbio su eventuali risvolti sentimentali e affettivi di un incontro solamente erotico — «si potrebbe trattare di bisogno d'amore/ meglio non dire» - e assume la sua libera scelta: «vorrei/ vorrei/ e lei adesso sa che vorrei/ le mani le sue/ [...] vorrei per amore o per ridere/ dipende da me».

\title{
Conclusione
}

\author{
Ma che politica, che cultura, \\ sono solo canzonette!... \\ Edoardo Bennato, Sono solo canzonette, 1980
}

In effetti, le canzoni studiate sembrano in décalage con la tormentata realtà degli anni Settanta. Raccontano essenzialmente storie d'amore con rarissimi riferimenti diretti alla vita sociopolitica italiana. Tuttavia, la canzone leggera di quegli anni comincia a parlare una lingua più vicina al quotidiano e, anche se le istanze femministe non riescono a incidere seriamente sulla sua scrittura, essa mostra talvolta segni d'emancipazione nelle immagini delle figure femminili proposte. Abbiamo visto che vi predominano stereotipi sulle figure dell'uomo e della donna, ma abbiamo anche scoperto una nuova sensibilità che riflette i dibattiti in voga sulla ridefinizione dei ruoli all'interno della coppia, e, più in generale, all'interno della società. Progressivamente le canzoni parlano sempre più esplicitamente di sesso, e, a differenza dei periodi precedenti, i personaggi femminili delle storie cantate rivendicano il diritto a vivere liberamente la propria sessualità come i loro partner maschili. L'interpretazione stessa delle cantanti diventa più sensuale e provocatoria.

Molte di queste canzoni, immortalate nella memoria di chi ha vissuto quel periodo, continuano a essere cantate ancora oggi. I ritmi e gli strumenti musicali, le modalità di esecuzione, gli interpreti possono variare, 
ma non le parole. Come può oggi interpretare questi testi un pubblico giovane che vive in una società che è cambiata rispetto a trenta-quarant'anni fa? Forse proprio perché versi e note formano un tutt'uno, forse perché il potere della musica supera quello delle parole, alcune di queste canzoni hanno sempre un grande successo e riescono così a veicolare, in modo subliminale e surrettizio, immagini e concetti superati... purtroppo non ancora da tutti.

\section{Bibliografia}

Antonelli Giuseppe, Ma cosa vuoi che sia una canzone, Bologna, Il Mulino, 20Io.

Berselli Edmondo, Canzoni. Storie dell'Italia leggera, Bologna, Il Mulino, 2007.

Borgna Gianni, Storia della canzone italiana, Milano, Mondadori, I992.

-, L'Italia di Sanremo. Cinquant'anni di canzoni, cinquant'anni della nostra storia, Milano, Mondadori, 1998.

Cartago Gabriella, La lingua della canzone, in La lingua italiana e i mass media, a cura di Ilaria Bonomi, Andrea Masini, Silvia Morgana, Roma, Carocci, 2002, pp. 199-222.

Cortellazzo Michele, La lingua della canzone d'autore nella storia dellitaliano contemporaneo, in Tradurre la canzone d'autore, Atti del Convegno, Milano, Università Bocconi, 29 settembre 1997, a cura di Giuliana Garzone, Leandro Schena, Bologna, CLUEB, 2000, pp. 2I-34.

Cover Lorenzo, Per una storia linguistica della canzone italiana, in Parole in musica. Lingua e poesia nella canzone d'autore italiana, a cura di Lorenzo Coveri, Novara, Interlinea, 1996, pp. 13-24.

Dall'Orto Giovanni e Casi Stefano, Il gay canzonato - L'omosessualità nelle canzoni italiane, in "Babilonia», 39, settembre 1986, pp. 48-52.

De Luigi Mario, Cultura e canzonette, Milano, Gammalibri, I980.

Di Franco Giovanni e Nobile Stefano, L'Italia che si dispera e l'Italia che si innamora. Temi, valori e linguaggi in 25 anni di canzone italiana, Roma, Paper, 1995.

FAB bri Franco, Il suono in cui viviamo, Milano, Il Saggiatore, 2008.

-, Around the clock, Torino, UTET, 2008.

Franco-Lao Meri, Donna canzonata, Roma, Newton Compton, I979.

Fratarcangeli Fernando, Patty Pravo, Roma, Coniglio, 2007.

Gatto Paolo, Il sesso, la donna, l'amore nella canzone italiana, Firenze,

D’Anna, 1977. 
Gentile Enzo, Legata a un granello di sabbia, Milano, Melampo, 2005. Guichard Jean, Il Pinocchio cantato: canzoni per un burattino, in Pinocchio esportazione: il burattino di Collodi nella critica straniera, a cura di Giorgio Cusatelli, Roma, Armando, 2002, pp. I29-I46.

Infanti Anna, La lingua della canzone, in "Quaderni dell'osservatorio linguistico", I, 2002, pp. 133-I60.

Io canto la differenza. Canzoni di donne e sulle donne, a cura di Maria Grazia Caldirola, Milano, Mazzotta, 1977.

JaCHia Paolo, La canzone d'autore italiana 1958-1997, Milano, Feltrinelli, 1998.

Liperi Felice, Storia della canzone italiana, Roma, Rai, Eri, I999.

Manfredi Gianfranco, Lucio Battisti. Canzoni e spartiti, Roma, Lato Side, 1979.

Mina, una forza incantatrice, a cura di Franco Fabbri e Luigi Pestalozza, Milano, Euresis, 1998.

Padovano Romy, Hit Parade. Classifiche, dischi, artisti dagli anni 'so ai nostri giorni, Milano, Mondadori, 1997.

—, Mina. I mille volti di una voce, Milano, Mondadori, 1998.

Peroni Marco, Il nostro concerto: la storia contemporanea tra musica leggera e canzone popolare, Milano, Mondadori, 2005.

Pivato Stefano, La storia leggera. L'uso pubblico della storia nella canzone italiana, Bologna, Il Mulino, 2002.

Pivato Stefano e Tonelli Anna, Italia vagabonda, Roma, Carocci, 200 I.

Salvatore Gianfranco, Mogol-Battisti. L'alchimia del verso cantato. Arte e linguaggio della canzone moderna, Roma, Castelvecchi, 1997.

Salvadori Dario, 25 anni di Hit Parade in Italia, Milano, Mondadori, I982.

Tu musica divina. Canzoni e storia in cento anni d'Italia, a cura di Giancarlo Governi, Franco Lefevre, Claudia Terenzi, Torino, Allemandi, 1997. 


\section{Allegati}

\section{Allegato I}

Corpus (secondo l'ordine di presentazione nell'articolo):

I. Tanta voglia di lei-Pooh

2. Anna-Lucio Battisti

3. Sotto il segno dei pesci - Antonello Venditti

4. Ti amo - Umberto Tozzi

5. La canzone del sole - Lucio Battisti

6. La fata-Edoardo Bennato

7. Anche per te-Lucio Battisti

8. Sara-Antonello Venditti

9. Sono una donna, non sono una santa - Rosanna Fratello

Io. Ragazza del Sud - Gilda

II. Finché la barca va-Orietta Berti

I2. Insieme - Mina

13. Grande grande grande - Mina

14. L'importante è finire - Mina

15. America - Gianna Nannini

16. Piccola storia ignobile - Francesco Guccini

17. Morta per autoprocurato aborto - Gianna Nannini

I8. Volo $A Z 504$ - Albatros

19. Gli occhi verdi di tua madre - Sandro Giacobbe

20. Pensiero Stupendo - Patty Pravo

2I. Il triangolo no - Renato Zero

Altre canzoni citate:

I. Cantautore - Edoardo Bennato

2. L'avvelenata - Francesco Guccini

3. Comunque bella - Lucio Battisti

4. Caffe nero bollente - Fiorella Mannoia

5. Disperato Erotico Stomp - Lucio Dalla

6. La fabbricante d'angeli - Le Orme

7. Andrea-Fabrizio De André

8. Lei-Gianna Nannini

9. Giulia - Antonello Venditti

Io. Tu che sei mio fratello - Renato Zero

II. Pierre - Pooh

I2. Sono solo canzonette - Edoardo Bennato 


\section{LiCIA BAGINI}

\section{Allegato 2}

\section{I97I, Pooh - Tanta voglia di lei}

Mi dispiace di svegliarti forse un uomo non sarò ma ad un tratto so che devo lasciarti fra un minuto me ne andrò. E non dici una parola sei più piccola che mai in silenzio morderai le lenzuola so che non perdonerai.

Mi dispiace devo andare il mio posto è là

il mio amore si potrebbe svegliare chi la scalderà.

Strana amica di una sera io ringrazierò la tua pelle sconosciuta e sincera ma nella mente c'è tanta tanta voglia di lei.

\section{0, Lucio Battisti - Anna}

Hai ragione anche tu cosa voglio di più un lavoro io l'ho una casa io l'ho la mattina c'è chi mi prepara il caffè questo io lo so e la sera c’è chi non sa dir di no cosa voglio di più hai ragione tu cosa voglio di più cosa voglio

Anna voglio Anna.

Non hai mai visto un uomo piangere apri bene gli occhi sai perché tu ora lo vedrai apri bene gli occhi sai perché tu ora lo vedrai se tu...

\author{
Lei si muove e la sua mano \\ dolcemente cerca me \\ e nel sonno \\ sta abbracciando pian piano \\ il suo uomo che non c'è. \\ Mi dispiace devo andare \\ il mio posto è là \\ il mio amore si potrebbe svegliare \\ chi la scalderà... \\ nella mente c'è tanta, \\ tanta voglia di lei. \\ Chiudo gli occhi un solo istante \\ la tua porta è chiusa già \\ ho capito che cos'era importante \\ il mio posto è solo là.
}

non hai mai visto un uomo piangere... guardami... guardami...

Anna...

voglio Anna.

Ho dormito lì

fra i capelli suoi

io insieme a lei

ero un uomo.

Quanti e quanti sì

ha gridato lei

quanti non lo sai

ero un uomo.

Cosa sono ora io?

Cosa sono mio Dio?

Resta poco di me

io che parlo con te

io che parlo con te

di...

Anna

Anna

voglio Anna

voglio Anna... 


\section{8, Antonello Venditti - Sotto il segno dei pesci}

Ti ricordi quella strada, eravamo io e te, e la gente che correva, e gridava insieme a noi, tutto quello che voglio, pensavo, è solamente amore, ed unità per noi, che meritiamo un'altra vita più giusta e libera se vuoi, corri amore, corri non aver paura.

Mi chiedevi che ti manca, una casa tu ce l'hai, hai una donna, una famiglia, che ti tira fuori dai guai, ma tutto quello che voglio, pensavo, è solamente amore,

ed unità per noi, che meritiamo un'altra vita più giusta e libera se vuoi, nata sotto il segno, nata sotto il segno dei pesci.
Ed il rock passava lento sulle nostre discussioni, I8 anni son pochi, per promettersi il futuro, ma tutto quel che voglio, dicevo, è solamente amore, ed unità per noi che meritiamo un'altra vita, violenta e tenera se vuoi, nata sotto il segno, nata sotto il segno dei pesci. E Marisa se n'è andata, oggi insegna in una scuola, vive male e insoddisfatta, e capisce perché è sola, ma tutto quel che cerca e che vuole è solamente amore ed unità per noi, che meritiamo un'altra vita, violenta e tenera se vuoi, nata sotto il segno, nata sotto il segno dei pesci. E Giovanni è un ingegnere che lavora in una radio, ha bruciato la sua laurea, vive solo di parole ma tutto quel che cerca e che vuole è solamente amore ed unità per noi, stretti in una libera sorte, violenti e teneri se vuoi figli di una vecchia canzone. 


\section{LiCIA BAGINI}

\section{7, Umberto Tozzi - Ti amo}

Ti amo,

un soldo

ti amo,

in aria

ti amo

se viene testa vuol dire che basta

lasciamoci.

Ti amo, io sono, ti amo, in fondo un uomo che non ha freddo nel cuore, nel letto

comando io

ma tremo davanti al tuo seno,

ti odio e ti amo,

è una farfalla che muore sbattendo le ali

l'amore che a letto si fa

rendimi l'altra metà

oggi ritorno da lei

primo Maggio, su coraggio.

Io ti amo e chiedo perdono

ricordi chi sono

apri la porta a un guerriero di carta igienica

e dammi il tuo vino leggero

che hai fatto quando non c'ero

e le lenzuola di lino

dammi il sonno di un bambino

che fa

sogna

cavalli e si gira

e un po' di lavoro fammi abbracciare una donna che stira

cantando

e poi fatti un po' prendere in giro

prima di fare l'amore

vesti la rabbia di pace e sottane sulla luce

io ti amo e chiedo perdono

ricordi chi sono

ti amo, ti amo, ti amo ti amo

e dammi il tuo vino leggero

che hai fatto quando non c'ero

e le lenzuola di lino

dammi il sonno di un bambino

che fa

sogna

cavalli e si gira

e un po' di lavoro

fammi abbracciare una donna che stira

cantando

e poi fatti un po' prendere in giro

prima di fare l'amore

vesti la rabbia di pace e sottane sulla luce

io ti amo e chiedo perdono

ricordi chi sono

ti amo, ti amo, ti amo ti amo... 


\section{2, Lucio Battisti - La canzone del sole}

Le bionde trecce gli occhi azzurri e poi

le tue calzette rosse

e l'innocenza sulle gote tue

due arance ancor più rosse

e la cantina buia dove noi

respiravamo piano

e le tue corse, l'eco dei tuoi no, oh no

mi stai facendo paura.

Dove sei stata cos'hai fatto mai?

Una donna, donna dimmi

cosa vuol dir sono una donna ormai.

Ma quante braccia ti hanno stretto, tu lo sai

per diventar quel che sei

un fiore in bocca può servire, sai

più allegro tutto sembra

e d'improvviso quel silenzio fra noi

e quel tuo sguardo strano

ti cade il fiore dalla bocca e poi

oh no, ferma, ti prego, la mano.

Dove sei stata cos' hai fatto mai?

Una donna, donna, donna dimmi

cosa vuol dir sono una donna ormai.

Io non conosco quel sorriso sicuro che hai non so chi sei, non so più chi sei

mi fai paura oramai, purtroppo.

Ma ti ricordi le onde grandi e noi

che importa tanto tu non me lo dirai, purtroppo. gli spruzzi e le tue risa

Ma ti ricordi l'acqua verde e noi

le rocce, bianco il fondo

di che colore sono gli occhi tuoi

se me lo chiedi non rispondo.

O mare nero, o mare nero, o mare ne...

tu eri chiaro e trasparente come me

o mare nero, o mare nero, o mare ne...

tu eri chiaro e trasparente come me.

Le biciclette abbandonate sopra il prato e poi cos'è rimasto in fondo agli occhi tuoi

la fiamma è spenta o è accesa?

O mare nero, o mare nero, o mare ne...

tu eri chiaro e trasparente come me

o mare nero, o mare nero, o mare ne...

tu eri chiaro e trasparente come me.

Il sole quando sorge, sorge piano e poi

la luce si diffonde tutto intorno a noi

noi due distesi all'ombra

le ombre ed i fantasmi della notte sono alberi

e cespugli ancora in fiore

sono gli occhi di una donna

ancora pieni d'amore. 


\section{LiCIA BAGINI}

\section{7, Edoardo Bennato - La fata}

C'è solo un fiore in quella stanza

ma da sempre

e tu ti muovi con pazienza

la medicina è amara ma

tu già lo sai che la berrà.

tu sei quella che paga di più

se vuoi volare ti tirano giù

e se comincia la caccia alla streghe

la strega sei tu.

Se non si arrende tu lo tenti

e sciogli il nodo dei tuoi fianchi

E insegui sogni da bambina

e quel vestito scopre già

e chiedi amore e sei sincera

chi coglie il fiore impazzirà.

non fai magie, né trucchi, ma

nessuno ormai ci crederà.

Farà per te qualunque cosa

e tu sorella e madre e sposa

C'è chi ti urla che sei bella

e tu regina o fata, tu

non puoi pretendere di più.

che sei una fata, sei una stella

poi ti fa schiava, però no

chiamarlo amore non si può.

E forse è per vendetta

e forse è per paura

C'è chi ti esalta, chi ti adula

o solo per pazzia

c'è chi ti espone anche in vetrina

si dice amore, però no

chiamarlo amore non si può.

\section{I97I, Lucio Battisti - Anche per te}

Per te che è ancora notte e già prepari il tuo caffè che ti vesti senza più guardar lo specchio dietro te che poi entri in chiesa e preghi piano

Per te che di mattina svegli il tuo bambino e poi lo vesti e lo accompagni a scuola e al tuo lavoro vai per te che un errore ti è costato tanto

e intanto pensi al mondo ormai per te così lontano. Per te che di mattina torni a casa tua perché per strada più nessuno ha freddo e cerca più di te per te che metti i soldi accanto a lui che dorme e aggiungi ancora un po' d'amore a chi non sa che farne.

Anche per te vorrei morire ed io morir non so anche per te darei qualcosa che non ho e così, e così, e così che tremi nel guardare un uomo e vivi di rimpianto. Anche per te vorrei morire ed io morir non so anche per te darei qualcosa che non ho e così, e così, e così io resto qui a darle i miei pensieri, a darle quel che ieri avrei affidato al vento cercando di raggiungere chi... al vento avrebbe detto sì.

io resto qui

a darle i miei pensieri,

a darle quel che ieri

avrei affidato al vento cercando di raggiungere chi... al vento avrebbe detto sì. 


\section{8, Antonello Venditti - Sara}

Sara, svegliati è primavera.

Sara, sono le sette e tu devi andare a scuola, Sara, prendi tutti i libri e accendi il motorino e poi attenta, ricordati che aspetti un bambino. Sara, se avessi i soldi ti porterei ogni giorno al mare, Sara, se avessi tempo ti porterei ogni giorno a far l'amore, ma Sara, mi devo laureare, e forse un giorno ti sposerò, magari in chiesa, dove tua madre sta aspettando per poter piangere un po'

Sara, tu va dritta non ti devi vergognare,

le tue amiche dai retta a me lasciale tutte parlare, Sara, è stato solo amore, se nel banco non c'entri più, tu sei bella, anche se i vestiti non ti stanno più. Sara, mentre dormivi l'ho sentito respirare, Sara, mentre dormivi ti batteva forte il cuore, Sara, tu non sei più sola, il tuo amore gli basterà, il tuo bambino, se ci credi nascerà Sara, Sara, Sara...

\section{2, Rosanna Fratello - Sono una donna, non sono una santa}

Sono una donna non sono una santa Non tentarmi non sono una santa Non mi portare nel bosco di sera Ho paura nel bosco di sera.

Fra tre mesi, te lo prometto Che il mio amore tu l'avrai Ti assicuro, non è un dispetto, ogni cosa a suo tempo, lo sai.

Tre mesi sono lunghi da passare Quando l'amore stuzzica il tuo cuore Ti prego amore mio non mi lasciare Se non avessi te meglio morire.
Non sono sola, ho quattro fratelli Non scordare che ho quattro fratelli Dove sei stata, la gente ti guarda Resta in casa, la gente ti guarda.

Fra tre mesi saremo in maggio E il mio amore ti darò Gesù mio dammi coraggio Di resistere e dirgli di no.

Tre mesi sono lunghi da passare Quando l'amore stuzzica il tuo cuore Ti prego amore mio non mi lasciare Se non avessi te meglio morire.

Sono una donna non sono una santa Non tentarmi non sono una santa. 


\section{LiCIA BAGINI}

\section{5, Gilda - Ragazza del Sud}

Ragazza che ti affretti perché suona la messa, cammini a testa bassa, sorridi a chi conosci. Sei nata in un paese di aranci e oleandri, ti hanno insegnato a credere, a vivere aspettando... lui... La treccia non la tagli, sarà il tuo primo amore a scioglierti i capelli e allora tremerai.

Ragazza del Sud, tu che sogni il mio mondo, guarda solo il mio viso, ragazza del Sud.

Al di sotto di quello che nasconde il sorriso, guarda in fondo, più in fondo: cento giorni di vita, mille anni di vita, il primo bacio l'ho avuto, non ricordo da chi. Il mio tempo non conta, no, ragazza del Sud.

\section{0, Orietta Berti - Finché la barca va}

Il grillo disse un giorno alla formica: «Il pane per l'inverno tu ce l'hai! Perché protesti sempre per il vino? Aspetta la vendemmia e ce l'avrai.» Mi sembra di sentire mio fratello che aveva un grattacielo nel Perù,

Voleva arrivare fino in cielo e il grattacielo adesso non l'ha più.

Finché la barca va lasciala andare, Finché la barca va tu non remare, Finché la barca stai a guardare, Quando l'amore viene il campanello suonerà, Quando l'amore viene il campanello suonerà. E tu che vivi sempre sotto il sole, Tra file di ginestri e di lillà.
Rimani a ricamare

il tuo nome sul lenzuolo, spiando alla finestra col primo batticuore. Ripartirò sognando forse un poco anch'io, sempre ch'io sappia ancora come si fa a sognare.

Ragazza del Sud, mi chiami signora e guardi ammirata i vestiti che ho.

Ragazza del Sud, darei tutto quanto per avere il tuo sguardo: cento giorni di vita, mille anni di vita, ho gli stessi tuoi anni, ma da quando non so.

Il mio tempo non conta, no, ragazza del Sud.

Al tuo paese c'è chi ti vuol bene Perché sogni le donne di città? Mi sembra di vedere mia sorella che aveva un fidanzato di Cantù,

Voleva averne uno anche in Cina e il fidanzato adesso non l'ha più.

Ritornello.

Stasera mi è suonato il campanello, è strano io l'amore ce l'ho già. Vorrei aprire in fretta il mio cancello, mi fa morire la curiosità.

Ma il grillo disse un giorno alla formica: «Il pane per l'inverno tu ce l'hai»; Vorrei aprire in fretta il mio cancello, ma quel cancello io non l'apro mai!

Ritornello. (x3) 
1970, Mina - Insieme

Io non ti conosco

io non so chi sei

so che hai cancellato con un gesto

i sogni miei

sono nata ieri nei pensieri tuoi

eppure adesso siamo insieme

non ti chiedo sai quanto resterai

dura un giorno la mia vita

io saprò che l'ho vissuta

anche solo un giorno

ma l'avrò fermata insieme a te

a te, a te, che ormai sei mio

tu l'amore io

insieme, insieme

la, la, la, la, la

io ti amo e ti amerò

finché lo vuoi

anche sempre se tu lo vorrai

insieme, insieme, insieme a te

la, la, la, la, la, la, la, la,

\section{2, Mina - Grande grande grande}

Con te dovrò combattere

non ti si può pigliare come sei

i tuoi difetti son talmente tanti

che nemmeno tu li sai.

Sei peggio di un bambino capriccioso

la vuoi sempre vinta tu,

sei l'uomo più egoista e prepotente

che abbia conosciuto mai.

Ma c'è di buono che al momento giusto

tu sai diventare un altro,

in un attimo tu

sei grande, grande, grande, le mie pene

non me le ricordo più.

Io vedo tutte quante le mie amiche

son tranquille più di me,

non devono discutere ogni cosa

come tu fai fare a me, tu l'amore io

insieme, insieme

io non ti conosco

io non so chi sei

so che hai cancellato con un gesto

i sogni miei

sono nata ieri nei pensieri tuoi

eppure adesso siamo insieme

non ti chiedo sai quanto resterai

dura un giorno la mia vita

io saprò che l'ho vissuta

anche solo un giorno

ma l'avrò fermata insieme a te

a te che ormai sei mio

tu l'amore io

insieme, insieme

la, la, la, la, la

tu l'amore io

insieme, insieme... ah. ricevono regali e rose rosse

per il loro compleanno

dicon sempre di sì

non hanno mai problemi e son convinte

che la vita è tutta lì.

Invece no, invece no

la vita è quella che tu dai a me,

in guerra tutti i giorni sono viva

sono come piace a te.

Ti odio poi ti amo poi ti amo, poi ti odio, poi ti amo,

non lasciarmi mai più

sei grande, grande, grande

come te sei grande solamente tu.

Ti odio poi ti amo poi ti amo, poi ti odio poi ti amo

non lasciarmi mai più

sei grande, grande, grande

come te sei grande solamente tu. 
1975, Mina - L'importante è finire

Adesso arriva lui, apre piano la porta

poi si butta sul letto

e poi e poi

ad un tratto io sento

afferrarmi le mani

le mie gambe tremare

e poi e poi e poi e poi

spegne adagio la luce,

la sua bocca sul collo

ha il respiro un po' caldo

ho deciso lo mollo,

ma non so se poi farlo

o lasciarlo soffrire

l'importante è... finire.

\section{9, Gianna Nannini - America}

Cercherò mi sono sempre detta cercherò troverai mi hanno sempre detto troverai per oggi sto con me mi basto nessuno mi vede

e allora accarezzo la mia solitudine

ed ognuno ha il suo corpo a cui sa cosa chiedere chiedere chiedere chiedere...

Fammi sognare lei si morde la bocca e si sente l'America

Fammi volare lui allunga la mano e si tocca

l'America

Fammi l'amore forte sempre più forte come fosse

l'America

Fammi l'amore forte sempre più forte ed io sono

l'America...

Cercherai mi hanno sempre detto cercherai e troverò ora che ti accarezzo troverò

ma quanta fantasia ci vuole per sentirsi in due

quando ognuno è da sempre nella sua solitudine e regala il suo corpo ma non sa cosa chiedere

chiedere chiedere chiedere...

Fammi volare lei le mani sui fianchi come fosse

l'America

Fammi sognare lui che scende e che sale e si sente

l'America

Fammi l'Amore lei che pensa ad un altro e si

inventa l'America

Fammi l'amore forte sempre più forte ed io sono

l'America...
Adesso volta la faccia,

questa è l'ultima volta che lo lascio morire

e poi e poi

ha talento da grande lui

nel fare l'amore

sa pigliare il mio cuore

e poi e poi e poi e poi

ha il volto sconvolto

io gli dico ti amo,

ricomincia da capo

è violento il respiro,

io non so se restare

o rifarlo morire

l'importante è... finire.

\section{6, Gianna Nannini - \\ Morta per autoprocurato aborto}

La stanza tua piena di fiori

e due coltelli i testimoni di un rito che non ha padroni un rito l'unico rimedio a libertà negate a volontà

spezzate

in mezzo al sangue lei per terra vinceva la sua guerra senza parlare senza accusare dei suoi tre mesi

di dolore di rancore di timore

ecco l'immagine e tutto a un tratto mi sembra assurdo le strade son di burro si scivolava si sprofondava che si faceva noi...

Dov'è il coraggio di continuare a dar la vita tra le macerie se la gente non ci sente più forse daranno un paradiso a donne come lei che così han deciso e in tutta questa distruzione io cerco un'altra direzione ma sono già troppo lontana quel coso brucia dentro me dentro di me dentro di me si torce l'anima cos'è successo che cosa resta adesso che cosa suono io le grida spaesate le mani morsicate sue... 


\section{6, Francesco Guccini - Piccola storia ignobile}

Ma che piccola storia ignobile mi tocca raccontare, così solita $\mathrm{e}$ banale come tante,

che non merita nemmeno due colonne su un giornale o una musica o parole un po' rimate,

che non merita nemmeno l'attenzione della gente, quante cose più importanti hanno da fare, se tu te la sei voluta, a loro non importa niente, te l'avevan detto che finivi male...

Ma se tuo padre sapesse qual è stata la tua colpa rimarrebbe sopraffatto dal dolore, uno che poteva dire «guardo tutti a testa alta», immaginasse appena il disonore,

lui che quando tu sei nata mise via quella bottiglia per aprirla il giorno del tuo matrimonio,

ti sognava laureata, era fiero di sua figlia, se solo immaginasse la vergogna, se solo immaginasse la vergogna, se solo immaginasse la vergogna...

E pensare a quel che ha fatto per la tua educazione, buone scuole e poca e giusta compagnia, allevata nei valori di famiglia e religione, di ubbidienza, castità e di cortesia, dimmi allora quel che hai fatto chi te l'ha mai messo in testa o dimmi dove e quando l'hai imparato che non hai mai visto in casa una cosa men che onesta e di certe cose non si è mai parlato

e di certe cose non si è mai parlato

e di certe cose non si è mai parlato...

E tua madre, che da madre qualche cosa l'ha intuita e sa leggere da madre ogni tuo sguardo: devi chiederle perdono, dire che ti sei pentita, che hai capito, che disprezzi quel tuo sbaglio.
Però come farai a dirle che nessuno ti ha costretta o dirle che provavi anche piacere, questo non potrà capirlo, perchè lei, da donna onesta, l'ha fatto quasi sempre per dovere, l'ha fatto quasi sempre per dovere, l'ha fatto quasi sempre per dovere...

E di lui non dire male, sei anche stata fortunata: in questi casi, sai, lo fanno in molti.

Sì, lo so, quando lo hai detto, come si usa, ti ha lasciata, ma ti ha trovato l'indirizzo e i soldi, poi ha ragione, non potevi dimostrare che era suo e poi non sei neanche minorenne ed allora questo sbaglio è stato proprio tutto tuo: noi non siamo perseguibili per legge, noi non siamo perseguibili per legge, noi non siamo perseguibili per legge...

E così ti sei trovata come a un tavolo di marmo desiderando quasi di morire, presa come un animale macellato stavi urlando, ma quasi l'urlo non sapeva uscire e così ti sei trovata fra paure e fra rimorsi davvero sola fra le mani altrui,

che pensavi nel sentire nella carne tua quei morsi di tuo padre, di tua madre e anche di lui, di tuo padre, di tua madre e anche di lui, di tuo padre, di tua madre e anche di lui?

Ma che piccola storia ignobile sei venuta a raccontarmi, non vedo proprio cosa posso fare. Dirti qualche frase usata per provare a consolarti o dirti: «È fatta ormai, non ci pensare». È una cosa che non serve a una canzone di successo, non vale due colonne su un giornale, se tu te la sei voluta cosa vuoi mai farci adesso e i politici han ben altro a cui pensare e i politici han ben altro a cui pensare e i politici han ben altro a cui pensare... 


\section{LiCIA BAGINI}

\section{6, Albatros - Volo AZ 504}

Ciao...

Ho passato dei bei giorni con te...

Piccolo, stupido, meraviglioso ragazzo.

Ciao... anzi addio...

Allora, non stavi scherzando,

te ne vai...

Certo che me ne vado...

cosa volevi

guarda quanto cielo c'è...

e quanti amori ci stanno dentro...

e quanti volano via.
Potevo lasciarti avere il bambino ma...

ti rendi conto, cosa sarebbe successo?

Però forse sarebbe stato meglio...

almeno non saresti andata via...

Sandra, ma dove vai?...

Dai non scherzare, torna qui...

Sandra, ascoltami, ma dai, cosa fai...

ti amo, Sandra...

ti amo, Sandra...

ti amo...

\section{6, Sandro Giacobbe - Gli occhi di tua madre}

Piove da qualche minuto

ti guardo e mi sento sfinito

mentre non riesco a spiegarti che cosa mi è capitato.

Era una sera normale

ti ero venuto a cercare

non c'eri però con tua madre mi misi a parlare

e quando l'ho avuta di fronte che scherzo

mi han fatto gli occhi miei

credevo che fossi tu ed era lei

poi mi sembrò naturale guardarla così come guardo te...

perché sei uscita, perché?

Mi hanno fatto innamorare

gli occhi verdi di tua madre

il sorriso di un tramonto

dove ci si può specchiare

i tuoi passi all'improvviso

e un tuffo al cuore immenso

... se ci penso...

la pioggia continua a cadere e tu continui

a non parlare hai l'aria di un cigno che muore, la vittima la sai fare

di certo non è quel sospiro che può cancellare quel che sei:

tu rimani tu, e lei è lei

non è colpa mia se mi piace

ogni cosa che rassomiglia a te

adesso hai capito perché...

Mi hanno fatto innamorare

gli occhi verdi di tua madre

il sorriso di un tramonto

dove ci si può specchiare

i tuoi passi all'improvviso

e un tuffo al cuore immenso...

Mi hanno fatto innamorare

gli occhi verdi di tua madre

un sorriso di un tramonto

dove ci si può specchiare

i tuoi passi all'improvviso

e un tuffo al cuore immenso

se ci penso...

se ci penso... 


\section{8, Patty Pravo - Pensiero stupendo}

E tu

E noi

E lei

Fra noi

Vorrei

Non so

Che lei

O no

Le mani

Le sue

Pensiero stupendo

Nasce un poco strisciando

Si potrebbe trattare di bisogno d'amore Meglio non dire.

E tu

E noi

E lei

Fra noi

Vorrei

Vorrei

E lei adesso sa che vorrei

Le mani le sue

Prima o poi

Poteva accadere sai

Si può scivolare se così si può dire questioni di cuore.
Pensiero stupendo

Nasce un poco strisciando

Si potrebbe trattare di bisogno d'amore Meglio non dire.

E tu

E noi

E lei

Fra noi

Vorrei

Vorrei

E lei adesso sa che vorrei

Le mani le sue

E poi un'altra volta noi due

Vorrei per amore o per ridere

Dipende da me

E tu ancora

E noi ancora

E lei un'altra volta fra noi

Le mani questa volta sei tu e lei

E lei a poco a poco di più, di più

Vicini per questioni di cuore

Se così si può dire.

E tu ancora

E noi ancora

E lei un'altra volta fra noi

Fra noi fra noi.

Pensiero stupendo

Nasce un poco strisciando

Si potrebbe trattare di bisogno d'amore Meglio non dire... 


\section{8, Renato Zero - Il triangolo no}

L'indirizzo ce l'ho, rintracciarti non è un problema, ti telefonerò, ti offrirò una serata strana,

il pretesto lo sai... quattro dischi e un po' di wiskey... wooooh! Sarò grande vedrai, fammi spazio e dopo mi dirai, $\mathrm{mmm} \mathrm{mmm} \mathrm{mmm}$...

che maschio sei... Lui chi è? Come mai l'hai portato con te? Il suo ruolo mi spieghi qual è?

Io volevo incontrarti da sola semmai, mentre lui, lui chi è, lui chi è,

già è difficile farlo con te... Mollalo!

Lui chi è? Lui cos'è? Lui com'è?

È distratto ma è certo di troppo... Mollalo!

Mi aspettavo lo sai, un rapporto un po' più normale, quale eventualità, trovarmi una collocazione, ora spiegami dai, l'atteggiamento che dovrò adottare... wooooh! mentre io rischierei di trovarmi al buio, tra le braccia lui... mmm mmm mm... non è il mio tipo!

\section{6, Edoardo Bennato - Cantautore}

Tu sei forte

tu sei bello

tu sei imbattibile

tu sei incorruttibile

tu sei un ah ah cantautore

tu sei saggio

tu porti la verità

tu non sei un comune mortale

a te non è concesso barare

tu sei un ah ah cantautore

tu sei un'anima eletta

tu non accetti compromessi

tu non puoi sbagliare

tu non devi lasciarti andare

perché sei un ah ah cantautore

no tu non puoi più lamentarti

che ti senti male

che ti scoppia la testa

e non ce la fai più a guidare

ma non farci ridere a dire

che anche un camionista si ferma

ogni tanto a riposare

perché tu a un camionista

non ti puoi paragonare

tu sei un ah ah cantautore
Lui chi è? Si potrebbe vedere... si potrebbe inventare... si potrebbe rubare... Lui chi è, lui chi è, lui chi è già è difficile farlo con te... Mollalo! Il triangolo no, non l'avevo considerato, d'accordo ci proverò, la geometria non è un reato, garantisci per lui, per questo amore un po' articolato...

mentre io rischierei, ma il triangolo io lo rifarei... perché no? Lo rifarei...

Lui chi è? Lui chi è?

Lui chi è? E si vedrà...

Lui è...

Lui chi è, lui chi è,

e loro, dico loro chi sono?

non li senti

trattenere il respiro

quando sei lì in alto

e cammini sul filo

qui nel grande circo

tu oramai sei il re

tu sei buono

tu sei vero

tu sei onesto

tu sei modesto

tu sei un ah ah cantautore

tu sei semplice

tu sei sicuro

tu sei generoso

tu sei valoroso

tu sei un ah ah cantautore

tu sei senza macchia

tu sei senza peccato

tu sei intoccabile

tu sei inattaccabile... 


\section{6, Francesco Guccini - L'avvelenata}

Ma s'io avessi previsto tutto questo, dati causa e pretesto, le attuali conclusioni

credete che per questi quattro soldi, questa gloria da stronzi, avrei scritto canzoni;

va beh, lo ammetto che mi son sbagliato e accetto il "crucifige» e così sia,

chiedo tempo, son della razza mia, per quanto grande sia, il primo che ha studiato...

Mio padre in fondo aveva anche ragione a dir che la pensione è davvero importante,

mia madre non aveva poi sbagliato a dir che un

laureato conta più d'un cantante:

giovane e ingenuo io ho perso la testa, sian stati i libri

o il mio provincialismo,

e un cazzo in culo e accuse d'arrivismo, dubbi di

qualunquismo, son quello che mi resta...

Voi critici, voi personaggi austeri, militanti severi,

chiedo scusa a vossìa,

però non ho mai detto che a canzoni si fan rivoluzioni,

si possa far poesia;

io canto quando posso, come posso, quando ne ho voglia senza applausi o fischi:

vendere o no non passa fra i miei rischi, non comprate i miei dischi e sputatemi addosso...

Secondo voi ma a me cosa mi frega di assumermi la bega di star quassù a cantare,

godo molto di più nell'ubriacarmi oppure $\mathrm{a}$

masturbarmi o, al limite, a scopare...

se son d'umore nero allora scrivo frugando dentro alle nostre miserie:

di solito ho da far cose più serie, costruire su macerie

o mantenermi vivo...

Io tutto, io niente, io stronzo, io ubriacone, io poeta, io buffone, io anarchico, io fascista, io ricco, io senza soldi, io radicale, io diverso ed io uguale, negro, ebreo, comunista!

Io frocio, io perché canto so imbarcare, io falso, io vero, io genio, io cretino,

io solo qui alle quattro del mattino, l'angoscia e un po' di vino, voglia di bestemmiare!

Secondo voi ma chi me lo fa fare di stare ad ascoltare chiunque ha un tiramento?

Ovvio, il medico dice «sei depresso», nemmeno dentro al cesso possiedo un mio momento.

Ed io che ho sempre detto che era un gioco sapere usare o no ad un certo metro:

compagni il gioco si fa peso e tetro, comprate il mio didietro, io lo vendo per poco!

Colleghi cantautori, eletta schiera, che si vende alla sera per un po' di milioni,

voi che siete capaci fate bene a aver le tasche piene e non solo i coglioni...

Che cosa posso dirvi? Andate e fate, tanto ci sarà sempre, lo sapete,

un musico fallito, un pio, un teorete, un Bertoncelli o un prete a sparare cazzate!

Ma s'io avessi previsto tutto questo, dati causa e pretesto, forse farei lo stesso,

mi piace far canzoni e bere vino, mi piace far casino, poi sono nato fesso

e quindi tiro avanti e non mi svesto dei panni che son solito portare:

ho tante cose ancora da raccontare per chi vuole ascoltare e a culo tutto il resto! 


\section{LiCIA BAGINI}

\section{2, Lucio Battisti - Comunque bella}

Tu vestita di fiori

o di fari in città

con la nebbia o i colori

cogliere le rose a piedi nudi e poi

con la sciarpa stretta al collo bianca come mai ma... eri bella bella

comunque bella.

Quando l'arcobaleno

era in fondo ai tuoi occhi

quando sotto al tuo seno

l'ira avvelenava il cuore tuo perché

tu vedevi un'altra donna avvicinarsi a me

prima ancora che io capissi e riscegliessi te tu... eri bella bella

comunque bella.

Anche quando un mattino tornasti vestita di pioggia con lo sguardo stravolto da una notte d'amore siediti qui

non ti chiedo perdono perché tu sei un uomo. Coi capelli bagnati - so che capirai...

Con quei segni sul viso - mi spiace da morire sai coi tuoi occhi arrossati

mentre tu mentivi e mi dicevi che

ancora più di prima tu amavi me

tu... eri bella bella

comunque bella... 


\section{I98I, Fiorella mannoia - Caffe nero bollente}

Ammazzo il tempo bevendo caffè nero bollente in questo nido scaldato già dal sole paziente ma tu che smetti alle tre poi torni a casa da me tu che non senti più niente mi avveleni la mente. Un filo azzurro di luce scappa dalle serrande e cerco invano qualcosa $\mathrm{da}$ inventare in mutande un'automobile passa e una mosca vola bassa $\mathrm{mi}$ ronza gira gira ma sbaglio la mira. Vorrei cercare qualche cosa da fare fuori

e camminare senza orgoglio darsi a un rubacuori.

Ma io come Giuda so vendermi nuda la strada conosco attirarti nel bosco attirarti nel bosco attirarti. Voci di strada all'orecchio tutto è poco eccitante in questo inverno colore caffè nero bollente ammazzo il tempo così ma scapperò via di qui da questa casa galera che mi fa prigioniera. Con gli occhi chiusi a mille miglia per conto mio odio la sveglia che mi sveglia oh mio Dio.
Ma io come Giuda

so vendermi nuda

da sola sul letto

mi abbraccio mi cucco

malinconico digiuno

senza nessuno.

Io non ho bisogno di te perché io non ho bisogno di te io non ho bisogno di te perché io non ho bisogno delle tue mani mi basto sola. E ammazzo il tempo bevendo caffè nero bollente in questo nido scaldato ormai da un sole paziente che brucia dentro di me che è forte come il caffè un pomeriggio così oh no non voglio star qui. E poi mi fermo per guardarmi un istante le smagliature della vita sono tante.

Un ballo in cucina e sono ancora bambina un pranzo da sposa e butterò giù qualcosa e questa voglia che non passa mentre dentro bussa. Io non ho bisogno di te perché io non ho bisogno di te Io non ho bisogno di te perché io non ho bisogno di te perché io non ho bisogno di te perché io non ho bisogno di te perché io non ho bisogno di te io non ho bisogno di te... 


\section{7, Lucio Dalla - Disperato Erotico Stomp}

Ti hanno vista bere a una fontana che non ero io ti hanno vista spogliata la mattina, birichina biricò. Mentre con me non ti spogliavi neanche la notte, ed eran botte, Dio, che botte

ti hanno visto alzare la sottana, la sottana fino al pelo. Che nero!

Poi mi hai detto "poveretto, il tuo sesso dallo al gabinetto»

te ne sei andata via con la tua amica, quella alta, grande fica.

Tutte e due a far qualcosa di importante, di unico e di grande

io sto sempre a casa, esco poco, penso solo e sto in mutande.

Penso a delusioni a grandi imprese a una Tailandese ma l'impresa eccezionale, dammi retta, è essere normale. Quindi, normalmente, sono uscito dopo una settimana non era tanto freddo, e normalmente ho incontrato una puttana.

A parte il vestito, i capelli, la pelliccia e lo stivale aveva dei problemi anche seri, e non ragionava male.

\section{4, Le Orme - La fabbricante d'angeli}

Dal più profondo buio della notte due occhi vuoti sfuggono le stelle.

La fabbricante d'angeli è già scesa ma incespica coi ferri arrugginiti.

Sul ventre già fiorito di una ingenua ragazzina la luna si ferisce passando i vetri rotti.
Non so se hai presente una puttana ottimista e di sinistra, non abbiamo fatto niente, ma son rimasto solo, solo come un deficiente.

Girando ancora un poco ho incontrato uno che si era perduto

gli ho detto che nel centro di Bologna non si perde neanche un bambino

mi guarda con la faccia un po' stravolta e mi dice «sono di Berlino».

Berlino, ci son stato con Bonetti, era un po' triste e molto grande

però mi sono rotto, torno a casa e mi rimetterò in mutande.

Prima di salir le scale mi son fermato a guardare una stella

sono molto preoccupato, il silenzio m'ingrossava la cappella.

Ho fatto le mie scale tre alla volta, mi son steso sul divano,

ho chiuso un poco gli occhi, e con dolcezza è partita la mia mano...

E d'improvviso l'aria si raffredda; sui tetti scuri argenta la rugiada; più non respira il seno è ormai di pietra che un dolce inganno un giorno aveva sciolto. Stanca è ormai la vecchia che si perde nella nebbia mentre le campane annunciano la festa.

(Grazie a Francesca per questo testo.) 


\section{8, Fabrizio De André - Andrea}

Andrea s'è perso s'è perso e non sa tornare Andrea s'è perso s'è perso e non saprà tornare Andrea aveva un amore Riccioli neri Andrea aveva un dolore Riccioli neri.

C'era scritto sul foglio ch'era morto sulla bandiera C'era scritto e la firma era d'oro era firma di re

Ucciso sui monti di Trento dalla mitraglia. Ucciso sui monti di Trento dalla mitraglia. Occhi di bosco contadino del regno profilo francese Occhi di bosco soldato del regno profilo francese
E Andrea l'ha perso ha perso l'amore la perla più rara E Andrea ha in bocca un dolore la perla più scura.

Andrea raccoglieva violette ai bordi del pozzo Andrea gettava Riccioli neri nel cerchio del pozzo Il secchio gli disse «Signore il pozzo è profondo più fondo del fondo degli occhi della Notte del Pianto.»

Lui disse «Mi basta mi basta che sia più profondo di me.» Lui disse «Mi basta mi basta che sia più profondo di me.» 


\section{9, Gianna Nannini - Lei}

Lei quella volta quando smise di parlare e restammo in silenzio ore e ore la sua pelle sconosciuta assomigliava un po' alla mia e profumava profumava di malinconia e la notte che scendeva non sembrava vera... lei solo lei gli occhi suoi come stelle sulle strade mi portavano a viaggiare viaggiavo ed ero vento sconfinavo le pareti nel silenzio ero notte sul suo seno e sfuggivano le mani senza più paura donna una donna donna una donna lui da dietro il suo bicchiere lui che riempie le mie sere donna una donna donna una donna non ho niente da lasciarti e continuo a ricordarti.

Poi la guardai si aggiustò i capelli e mi parlò di un figlio che non era nato e leggero nel ricordo qualche posto qualche strada di paese la voce di sua madre finalmente sorrideva alla sua maniera donna una donna donna una donna lui che ha voglia di ascoltare lui che riempie il mio bicchiere donna una donna donna una donna lui che è tutto lui che è niente lui che è sempre più importante donna una donna donna una donna non ho niente da lasciarti forse solo solo ricordarti...

\section{8, AntonelloVenditti - Giulia}

Giulia gli occhiali sul naso che sfiora la mente parla di uomini e donne come solo lei sa e la camera è bassa e la mano piano piano che scende trova la tua tenerezza e la sua verità Giulia ci sa fare

Giulia è intelligente

Giulia è qualcosa di più

Giulia ti accarezza

Giulia lotta insieme

Giulia parla anche per te... ohh

è Giulia che ti tocca

è Giulia che ti porta via da me...

dove il cammino è deserto e il deserto è confine calda la luce degli occhi ti guiderà mentre io dovrei essere il tuo, o il suo giustiziere scopro la dolce vittoria della sua crudeltà Giulia ci sa fare

Giulia è intelligente

Giulia è qualcosa di più

Giulia ti accarezza

accarezza la tua mente

Giulia lotta anche per te

è Giulia che ti tocca

è Giulia che ti porta

via da me...

Perché lei è solo la mia vita

lei è solo tutto il mondo

lei è solo una gran parte di me

lei è solo una bambina

lei è solo troppo bella

lei è solo troppo anche per te

lei è solo un po' confusa

e ti prego non portarla

via da me...

Giulia oh Giulia... 


\section{4, Renato Zero - Tu che sei mio fratello}

Se vuoi,

Resto qui.

Ricomincio insieme a te!

Vedrai, guarirai.

Ti aiuterò a rinascere, se vuoi, se vuoi...

Io e te,

Torneremo,

Quell'attimo smarrito tempo fa...

Noi, noi, noi.

Noi siamo forti, noi.

Abbiamo gli occhi chiari noi,

$\mathrm{E}$ un sogno in più.

Mai, mai, mai.

Non tradiremo, mai,

La verità!

Tu che sei mio fratello,

La mia donna, il mio dio.

Tu che vivi in silenzio,

Non scordare il nome mio!

Sono qui, quando dormi,

Se hai paura di te,

Quando come un bambino, tremerai, sono qui!

Lo so,

Che cos'hai...

Quei fantasmi intorno a te...

Non dire di no,

Domani sarà certo il giorno tuo, vedrai!
Andrai,

Forte e fiero.

Nel mondo che non ti ha creduto mai!

Noi, noi, noi.

Noi siamo forti, noi!

Abbiamo gli occhi chiari, noi

E un sogno, in più!

Mai, mai, mai,

Non tradiremo, mai,

La verità!

Tu che sei mio fratello,

la mia donna, il mio dio.

Tu che vivi in silenzio,

Non scordare, il nome mio.

Sono qui quando dormi,

Se hai paura di te,

Quando come un bambino, tremerai!

Ma che cos'hai, fratello!?

Tu non mi senti, più...

Sarà triste il risveglio,

Ora che non ci sei, tu...

Tu te ne vai, fratello...

Ti giuro, vincerò!

Quell'azzurro, del cielo,

Lo vedrai, negli occhi miei!
1976, Pooh - Pierre

Penso a te

nei tempi della scuola con noi

sottile pallido e un po' perso

tu già da noi così diverso

triste.

Penso a te

ricordo, si rideva tra noi

di quel tuo sguardo di bambina

di quella tua dolcezza strana

triste. Pierre ti ho rivisto questa sera e tu tu abbassi gli occhi

ti nascondi e poi

te ne vai.

Scusami se ti ho riconosciuto però sotto il trucco gli occhi sono i tuoi non ti arrendi a un corpo che non vuoi senti...

Pierre sono grande ed ho capito sai io ti rispetto resta quel che sei tu che puoi. 


\section{I980, Edoardo Bennato - Sono solo canzonette}

$\mathrm{Mi}$ ricordo che anni fa di sfuggita dentro un bar ho sentito un juke-box che suonava e nei sogni di bambino la chitarra era una spada e chi non ci credeva era un pirata! ... e la voglia di cantare e la voglia di volare forse mi è venuta proprio allora forse è stata una pazzia però è l'unica maniera di dire sempre quello che mi va!...

Non potrò mai diventare direttore generale delle poste o delle ferrovie non potrò mai far carriera nel giornale della sera anche perché finirei in galera! ... mai nessuno mi darà il suo voto per parlare o per decidere del suo futuro nella mia categoria è tutta gente poco seria di cui non ci si può fidare!...

Guarda invece che scienziati, che dottori, che avvocati, che folla di ministri e deputati! pensa che in questo momento proprio mentre io sto cantando stanno seriamente lavorando! ... per i dubbi e le domande che ti assillano la mente va da loro e non ti preoccupare sono a tua disposizione e sempre, senza esitazione loro ti risponderanno!...

... io di risposte non ne ho! io faccio solo rock'n' roll! ... se ti conviene bene io più di tanto non posso fare!...

Gli impresari di partito mi hanno fatto un altro invito e hanno detto che finisce male se non vado pure io al raduno generale della grande festa nazionale! ... hanno detto che non posso rifiutarmi proprio adesso che anche a loro devo il mio successo, che son pazzo ed incosciente sono un irriconoscente un sovversivo, un mezzo criminale!...

Ma che ci volete fare non vi sembrerò normale ma è l'istinto che mi fa volare! non c'è gioco né finzione perché l'unica illusione è quella della realtà, della ragione! ... però a quelli in malafede sempre a caccia delle streghe dico: no! non è una cosa seria! e così e se vi pare ma lasciatemi sfogare non mettetemi alle strette e con quanto fiato ho in gola vi urlerò: non c'è paura! ma che politica, che cultura, sono solo canzonette!... 